\title{
Theoretical modeling of airways pressure waveform for dual-controlled ventilation with physiological pattern and linear respiratory mechanics
}

\section{Francesco Montecchia}

Laboratorio Sperimentale Policentrico di Ingegneria Medica, Dipartimento di Ingegneria Civile, Università degli Studi di Roma "Tor Vergata", Rome, Italy.

Email:francesco.montecchia@uniroma2.it

Received 13 January 2011; revised 28 March 2011; accepted 7 April 2011.

\section{ABSTRACT}

The present paper describes the theoretical treatment performed for the geometrical optimization of advanced and improved-shape waveforms as airways pressure excitation for controlled breathings in dual-controlled ventilation applied to anaesthetized or severe brain injured patients, the respiratory mechanics of which can be assumed linear. Advanced means insensitive to patient breathing activity as well as to ventilator settings while improved-shape intends in comparison to conventional square waveform for a progressive approaching towards physiological transpulmonary pressure and respiratory airflow waveforms. Such functional features along with the best ventilation control for the specific therapeutic requirements of each patient can be achieved through the implementation of both diagnostic and compensation procedures effectively carried out by the Advance Lung Ventilation System (ALVS) already successfully tested for square waveform as airways pressure excitation. Triangular and trapezoidal waveforms have been considered as airways pressure excitation. The results shows that the latter fits completely the requirements for a physiological pattern of endoalveolar pressure and respiratory airflow waveforms, while the former exhibits a lower physiological behaviour but it is anyhow periodically recommended for performing adequately the powerful diagnostic procedure.

Keywords: Mathematical Modeling; Mechanical Ventilation; Controlled Breathing; Pressure and Airflow Waveforms; Respiratory Mechanics; Tidal and Minute Volumes

\section{INTRODUCTION}

The clinical applications of assisted/controlled ventilation are mainly devoted to patients treated with anaesthesia or in Intensive Care Units or affected by the respiratory insufficient syndrome [1-3].

When spontaneous breathing of such patients is absent or forbidden for the entire time of treatment, controlled ventilation is required. The respiratory pattern during controlled ventilation shows only controlled breathings, i.e. breathings for which the control of lung ventilation is completely carried out by an external ventilator, in series with time $[4,5]$.

Otherwise, when spontaneous breathing is present, even if partially in time or below the standard physiological level, assisted/controlled ventilation is recommended. Allowing the patient the possibility of spontaneous breathing at his will or capability, assisted/controlled ventilation is so called because, it includes all that modalities or techniques in which the ventilator supplies the patient with controlled breathing only after a long lasting interval of apnea (assisted ventilation) or at detection of a very weak effort of spontaneous breathing (triggered ventilation). The respiratory pattern during assisted/controlled ventilation shows both controlled and spontaneous breathings in random series with time [6-7].

The controlled breathings supplied to patient during controlled or assisted/controlled ventilation can be properly classified considering the primary physical parameters controlled during the inspiration by the ventilator irrespective of load (respiratory characteristics of patient) variations or fluctuations as well as ventilator settings $[8$, 9].

Volume-controlled ventilation (VCV) or pressurecontrolled ventilation (PCV) refer to different modalities in which during the inspiration the ventilator supplies the load (lungs) with the pre-established volume (tidal or 
minute volume) through the selected respiratory airflow waveform or applies to the load the pre-established airways pressure waveform, respectively $[8,9]$.

The historical background of both VCV and PCV as well as their advantages and disadvantages in different clinical applications of assisted/controlled ventilation have been extensively described elsewhere $[9,10]$. In summary, considering the higher physiological character along with lower level of intrinsic pathological risks and functional failure involved, nowadays PCV is certainly the most adopted in the clinical practice [9-12].

The functional disadvantages of PCV which does not provide for the control of lung volume (tidal or minute) has been overcome with the implementation of dualcontrolled ventilation (DCV), i.e. PCV with ensured tidal or minute volume $[13,14]$. In detail, DCV is an advanced form of PCV in which the magnitude of selected airways pressure waveform is automatically regulated by feedback control for delivering during the inspiration time either the tidal volume required or, considering the current breathing frequency, the minute volume preestablished $[9,15]$. This is the so called DCV "breath to breath" mode, representing the most diffused form of DCV in the clinical practice $[15,16]$. In a different way, the so called DCV "within a breath" mode is a DCV mode in which the ventilator switches from pressure to volume control in the middle of the breath $[15,16]$.

In most cases during assisted/controlled ventilation, the respiratory system of healthy anaesthetized or severe brain injured patients exhibits a steady and reproducible response to controlled breathings, if evaluated as a whole. Moreover, the breathing dynamics involved is considerably reduced on account of small tidal volume required. Therefore, the respiratory mechanics of such patients can be properly assumed steady and linear [9]. According to PCV excitation hypothesis along with to steady and linear respiratory mechanics assumption, only DCV "breath to breath" mode will be considered in the present work. Moreover, DCV "breath to breath" mode is perfectly compatible with the feedback control adopted for the ventilation process [9] which regulates the operative parameters only between different breathings evaluated as a whole, i.e. in steady conditions and does not within the transient time of each breath $[9,15,16]$.

Until today, PCV or DCV have been mainly implemented with square waveform as airways pressure excitation, i.e. two different constant levels of airways pressure applied to patient during both inspiration and expiration $[12,14,16]$. Such strong limitation in waveform modeling of airways pressure controlled by the ventilator, resulting from simplified hardware and software design, reduces drastically the functional versatility of the venti- lator performances.

Among the different systems proposed for removing this limitation [8,9,17-28] and thus for evaluating the effect of varying inspiratory airflow waveforms on clinical parameters of mechanically ventilated patients [2931], the Advanced Lung Ventilation System (ALVS) has been conceived and designed for the waveform optimization of airways pressure excitation when controlled breathings have to be apply during assisted/controlled ventilation to anaesthetized or severe brain injured patients, the respiratory mechanics of which can be assumed steady and linear [9,32-36]. The functional flexibility and versatility of ALVS are both extremely useful for the research activity with an optimal and advanced ventilator as well as for its laboratory and clinical development and testing [9].

The present work deals with the description of both theory and ALVS settings performed for modeling a more realistic approximation of airways pressure excitation to physiological transpulmonary pressure waveform. The optimization of such excitation for patient, i.e. airways pressure waveform, has been carried out in order to reach a more physiological reaction of patient, i.e. respiratory airflow and endoalveolar pressure waveforms.

\section{METHODS}

The optimization of controlled breathing during assisted/ controlled ventilation obtained by the functional features of ALVS, has been extensively reported and discussed elsewhere [9]. Concerning the controlled breathings applied to patient during assisted/controlled ventilation, in order to improve over the conventional PCV with ensured tidal or minute volume, i.e. dual-controlled ventilation (DCV), ALVS has been designed for performing two subsequent functional steps.

The first step consists in the optimization of the ventilation control with conventional square waveform as airways pressure excitation applied to patient. This result has been already reached by means of two effective functional procedures: The diagnostic and the compensation procedures.

The theoretic approach on which the optimization of the ventilation control as well as both the diagnostic and the compensation procedures found, have been extensively reported in a previous paper [9] in which the respiratory mechanics of considered patients, i.e. anaesthetized or severe brain injured patients, has been properly assumed linear. Moreover, the ventilation control works by feedback regulation acting after the acquisition of each controlled breathing accounted as a whole, i.e. in steady conditions.

The diagnostic procedure establishes the optimal time of both inspiration and expiration taking into account the 
current respiratory characteristics (airways resistance and lung compliance) of patient and his diagnostic evaluations. Practically, the procedure sets the time of both inspiration and expiration as about five times the current inspiratory and expiratory time constants, the determination of which, along with other useful diagnostic parameter, is obtained real-time by ALVS monitoring system [37-39]. The determination of both airways resistance and lung compliance of patient is currently performed by the diagnostic procedure with high accuracy and without any unfavourable deformation of respiratory pattern otherwise introduced with the required artificial respiratory airflow interruption [40,41]. The high accuracy results from the application of the compensation procedure, described as follows, since it allows the correct implementation of the results available from the theory developed assuming a real square waveform as airways pressure excitation [9,42-44].

The compensation procedure stabilizes the airflow across the external resistance which controls the airways pressure applied to patient during the whole respiratory time. The procedure is performed through the variation during both inspiration and expiration of ALVS generator's internal resistance around its steady equilibrium value assumed during apnea, according to the respiratory airflow waveform resulting from patient's breathing activity and characteristics. The determination of the respiratory airflow waveform is obtained real-time by ALVS monitoring system. In such a way, ALVS behaves like an ideal airways pressure generator, making possible a real square waveform as airways pressure excitation through a proper square waveform as external resistance of ALVS controlling the airways pressure applied to patient, eliminating the airways pressure distortion induced by the dependence on current value of load (airways resistance and lung compliance) and its variations.

The experimental results obtained by ALVS connected with a well suited and versatile lung simulator performing the implementation of both diagnostic and compensation procedure for advanced square waveform as airways pressure excitation are completely in agreement with the theoretical ones, showing clearly that the ventilation control optimization has been reached. In particular, concerning the lung volume control, the results point out that the tidal or minute volume are independent on airways resistance or lung compliance, respectively [9].

The last results are very interesting from both clinical and engineering point of view since an increase of airways resistance (obstructive process) or a reduction of lung compliance (restrictive process) does not affect the control of tidal or minute volume, respectively, avoiding a critical regulation of the airways pressure levels applied to patient.
The second step consists in the optimization of the ventilation control with waveforms of improved shapes as airways pressure excitation applied to patients considering their current clinical conditions and specific therapeutic requirements. Improved shapes means more realistic approximation of airways pressure waveform to physiological transpulmonary pressure waveform inducing a more physiological reaction of patient, i.e. respiratory airflow and endoalveolar pressure waveforms.

The implementation of the diagnostic procedure in these cases also ensures that the optimal time of both inspiration and expiration is retained taking anyhow into account the current respiratory characteristic of patient (airways resistance and lung compliance) and his diagnostic evaluations. Moreover, the implementation of the compensation procedure in these cases also, making the selected airways pressure waveform insensitive to patient's respiratory characteristics, allows any airways pressure waveform of clinical interest during both inspiration and expiration through an identical shape of external resistance waveform which controls the airways pressure applied to patient.

In the present work, from a theoretical point of view, two waveforms of increasing geometrical shape with respect to conventional square waveform have been considered as airways pressure excitation applied to patient: triangular and trapezoidal. Accordingly to the physiopathological and clinical condition of patients considered as well as to the physical characteristics of controlled breathings in assisted/controlled ventilation modalities, the theoretical treatment in both cases has been carried out evaluating each controlled breathing as a whole, i.e. in steady conditions and assuming linear the respiratory mechanics of patients [9,32-36].

\section{RESULTS}

\subsection{Advanced Square Waveform as Airways Pressure Excitation (AD_SQUARE)}

Figure 1 shows the airways $\left(p_{A W}(t)\right)$ and endoalveolar $\left(p_{E A}(t)\right)$ pressures $(p(t))$ as well as the respiratory airflow $\left(\phi_{R E S}(t)\right)$ as a function of time $(t)$ resulting from the application to patient of the advanced square waveform as airways pressure excitation (AD_SQUARE). The time, the variables and the parameters relative to inspiration and expiration will be denoted with the addition of a specific pedix character (i) and (e), respectively. As depicted in Figure 1(b), on account of their opposite directions, the inspiratory $\left(\phi_{I N S}\left(t_{i}\right)\right)$ and expiratory $\left(\phi_{E X \mathrm{P}}\left(t_{e}\right)\right)$ airflows are conventionally considered positive and negative quantities, respectively.

The most relevant results obtained in the previous work [9] are summed up as follows. The real time 


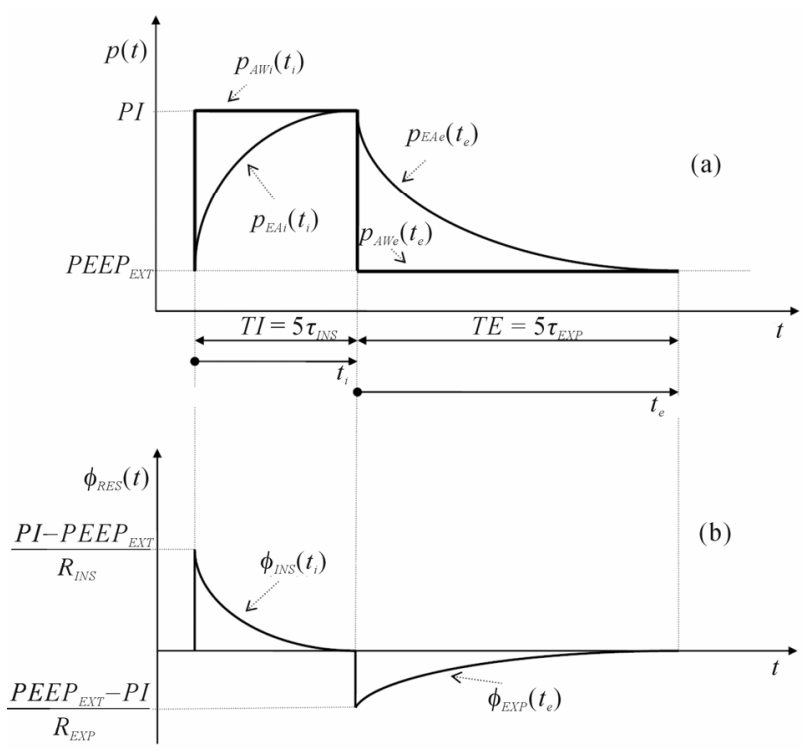

Figure 1. (a) Airways $\left(p_{A W}(t)\right)$ and endoalveolar $\left(p_{E A}(t)\right)$ pressures $(p(t))$ along with (b) respiratory airflow $\left(\phi_{R E S}(t)\right)$ as a function of time $(t)$ resulting from the application of $\mathrm{AD}_{-}$ SQUARE. In (b) inspiratory $\left(\phi_{I N S}\left(t_{i}\right)\right)$ and expiratory $\left(\phi_{E X P}\left(t_{e}\right)\right)$ airflows are depicted as positive and negative quantities, respectively, on account of their opposite directions.

monitoring of both $\phi_{I N S}\left(t_{i}\right)$ and $\phi_{E X P}\left(t_{e}\right)$ provides for the determination of the following parameters: $t_{T I} ; t_{T E}$; $\phi_{I N S}(0) ; \phi_{E X P}(0) . \phi_{I N S}(0)$ and $\phi_{E X P}(0)$ are the initial maximum values assumed by $\phi_{I N S}$ and $\phi_{E X P}$, respectively, while $t_{T I}$ and $t_{T E}$ are the times required for reaching the end of transient inspiration and expiration times, i.e. for observing a ninety nine percent $(99 \%)$ reduction of $\phi_{I N S}$ and $\phi_{E X P}$ with regard to $\phi_{I N S}(0)$ and $\phi_{E X P}(0)$, respectively.

If the upper $(P I)$ and lower or external positive end expiratory pressure $\left(P E E P_{E X T}\right)$ constant levels of square waveform as $p_{A W}$ excitation are kept for an inspiration $(T I)$ and expiration (TE) times equals to $t_{T I}$ and $t_{T E}$, respectively, the following expressions occur:

$$
\begin{gathered}
T I=t_{T I}=5 \tau_{I N S}=5 R_{I N S} C_{P} \\
T E=t_{T E}=5 \tau_{E X P}=5 R_{E X P} C_{P} \\
C_{P}=\frac{v_{p}(t)}{p_{E A}(t)} \\
p_{E A i}\left(t_{i}\right)=P I-\left(P I-P E E P_{E X T}\right) \mathrm{e}^{-\frac{t_{i}}{\tau_{I N S}}} \\
p_{E A e}\left(t_{e}\right)=P E E P_{E X T}+\left(P I-P E E P_{E X T}\right) \mathrm{e}^{-\frac{t_{e}}{\tau_{E X P}}} \\
p_{E A i}(T I)=P A P \cong p_{A W i}(T I)=P I \\
p_{E A e}(T E)=P E E P_{T O T} \cong p_{A W e}(T E)=P E E P_{E X T}
\end{gathered}
$$

$$
p_{E A i}(0)=p_{E A e}(T E) \cong P E E P_{E X T}
$$

$\tau_{I N S}$ and $\tau_{E X P}$ are the inspiratory and expiratory time constants, respectively, while $v_{P}(t)$ is the lung volume as a function of time.

According to the assumption of linear respiratory mechanics for controlled breathings accounted as a whole, i.e. in steady conditions [9], the static lung compliance $\left(C_{P}\right)$, defined by $(3)$, can be considered as constant during the whole respiration time, while the different values assumed by the respiratory airways resistance $\left(R_{R E S}\right)$ during inspiration $\left(R_{I N S}\right)$ and expiration $\left(R_{E X P}\right)$ can be both considered constant.

According to (6) and (7), the maximum or peak $(P A P)$ and minimum or total positive end expiratory pressure $\left(P E E P_{T O T}\right)$ values of $p_{E A}$ assumed at the end of inspiration $\left(t_{i}=T I\right)$ and expiration $\left(t_{e}=T E\right)$, respectively, can be easily detected since they equal the constant $P I$ and $P E E P_{E X T}$ values assumed by $p_{A W}$ during the inspiration $\left(0 \leq t_{i} \leq T I\right)$ and the expiration $\left(0 \leq t_{e} \leq\right.$ $T E$ ), respectively. (8) establishes that the value assumed by $p_{E A}$ at the beginning of inspiration $\left(p_{E A i}(0)\right)$ should be equal to that assumed at the end of last expiration $\left(p_{E A e}(T E)\right)$.

Concerning with $v_{P}$, considering (3), (6) and (8) at the beginning $\left(t_{i}=0\right)$ and end $\left(t_{i}=T I\right)$ of inspiration, the following expressions result:

$$
\begin{gathered}
v_{p i}(0)=C_{p} p_{E A i}(0) \cong C_{p} P E E P_{E X T}=F R C \\
v_{p i}(T I)=C_{p} p_{E A i}(T I) \cong C_{p} P I \\
V_{T I D}=v_{p i}(T I)-v_{p i}(0) \cong C_{p}\left(P I-P E E P_{E X T}\right)
\end{gathered}
$$

$F R C$ and $V_{\text {TID }}$ denote the functional residual capacity and the tidal volume delivered to patient for every inspiration.

If $T I$ and $T E$ are expressed in seconds, considering that the breathing period $(T R)$ equals to the sum $T I+T E$, from both (1) and (2), the breathing frequency $(F R)$, expressed in act for minutes, is defined as follows:

$$
F R=\frac{60}{T R}=\frac{60}{T I+T E}=\frac{60}{5 C_{P}\left(R_{I N S}+R_{E X P}\right)}
$$

Considering both (11) and (12), the so-called minute volume $\left(V_{M I N}\right)$, i.e. the volume delivered to patient for every minute, is given by the following expression:

$$
V_{M I N}=F R \times V_{T I D} \cong \frac{12\left(P I-P E E P_{E X T}\right)}{R_{I N S}+R_{E X P}}
$$

As pointed out in $\S 2,(11)$ and (13) establish that $V_{T I D}$ or $V_{M I N}$ are independent on $R_{R E S}$ (both $R_{I N S}$ and $R_{E X P}$ ) or $C_{P}$, respectively. That is extremely relevant from both clinical and engineering point of view since an increase 
of $R_{R E S}$ (obstructive process) or a reduction of $C_{P}$ (restrictive process) does not affect the control of $V_{T I D}$ or $V_{M I N}$, respectively, avoiding a critical regulation of $p_{A W}$ constant levels applied to patient.

As it is well known, the mean value $(M)$ of a periodic function of time $(t)$ with period $T\left(f_{T}(t)\right)$ is defined as follows:

$$
M=\frac{1}{T} \int_{0}^{T} f_{T}(t) \mathrm{d} t
$$

If $p_{A W}(t)$ and $p_{E A}(t)$ are considered as periodic functions of time with period $T R$, the mean $p_{A W}(t)(M A P)$ and $p_{E A}(t)$ $(M E P)$ assume the following expressions:

$$
\begin{aligned}
& M A P=\frac{1}{T R} \int_{0}^{T R} p_{A W}(t) \mathrm{d} t \\
& M E P=\frac{1}{T R} \int_{0}^{T R} p_{E A}(t) \mathrm{d} t
\end{aligned}
$$

From Figure 1(a), (1), (2), (4), (5), (12), (15) and (16), it is easy to demonstrate that $M A P$ and $M E P$ values of AD_SQUARE $\left(M A P_{\text {squ }}\right.$ and $\left.M E P_{\text {squ }}\right)$ result as follows:

$$
\begin{gathered}
M A P_{\text {squ }}=P E E P_{E X T}+\frac{\left(P I-P E E P_{E X T}\right) \tau_{I N S}}{\left(\tau_{I N S}+\tau_{E X P}\right)} \\
M E P_{\text {squ }} \cong P E E P_{E X T}+\frac{\left(P I-P E E P_{E X T}\right)\left(0,8 \tau_{I N S}+0,2 \tau_{E X P}\right)}{\left(\tau_{I N S}+\tau_{E X P}\right)}
\end{gathered}
$$

\subsection{Advanced Improved-Shape Waveforms as Airways Pressure Excitation}

The favourable results obtained with the implementation of AD_SQUARE in term of ventilation control optimization ( $(3.1)$, suggest theoretical effort for considering advanced and improved-shape waveforms as $p_{A W}$ excitation applied to patient. Advanced means insensitive to patient breathing activity as well as to ventilator settings. Improved-shape intends in comparison to conventional square waveform for a progressive approaching to physiological transpulmonary pressure waveform producing a more suitable reaction of patient, i.e. a more realistic approximation of $\phi_{R E S}$ and $p_{E A}$ waveforms to physiological ones.

For this reason, moving from AD_SQUARE, two waveforms of different geometrical shape which progressively approach the best solution are going to be considered: Triangular and trapezoidal. The problem to be solved consists in the proper smoothing of $\phi_{R E S}$ vertical discontinuities occurring at the beginning of both inspiration and expiration when $\phi_{R E S}$ is reversed, as response to upward and downward $p_{A W}$ vertical transitions characteristic of square waveform. So that, the elimina- tion of such $p_{A W}$ vertical transitions is the most relevant change to be applied on AD_SQUARE.

\subsection{Advanced Triangular Waveform as Airways Pressure Excitation (AD_TRIANG)}

Figure 2 shows the advanced triangular waveform as $p_{A W}$ excitation (AD_TRIANG). Unlike AD_SQUARE, where $p_{A W}$ is kept constant during the whole time of inspiration $\left(0 \leq t_{i} \leq T I\right)$, in AD_TRIANG $p_{A W}$ increases linearly from minimum or $P E E \bar{P}_{E X T}$ to maximum or peak $(P I P)$ values assumed at the beginning $\left(t_{i}=0\right)$ and at the end $\left(t_{i}=T I\right)$ of the time during inspiration $\left(t_{i}\right)$, respectively. The linear increase of $p_{A W}$ has been selected for smoothing $\phi_{R E S}$ discontinuity occurring at the beginning of every inspiration as response to upward $p_{A W}$ vertical transition of AD_SQUARE. As in AD_SQUARE, during the whole time of expiration $\left(0 \leq t_{e} \leq T E\right) p_{A W}$ is kept constant on PEEP $P_{E X T}$ value.

AD_TRIANG can be carried out by connecting the patient's airways with an ideal generator creating a triangular waveform of $p_{A W}$. The electrical-equivalent circuit of AD_TRIANG generator connected to the patient's airways is shown in Figure 3. The respiratory mechanics of patient (lung simulator) has been treated with a steady and linear physical model consisting of the respiratory airways resistance $\left(R_{R E S}\right)$ connected in series to the lung compliance $\left(C_{P}\right)[9,45,46]$. Such physical model does not include any inductance on account of negligible inertia of airflow as well as airways, lungs and chest tissues at very low breathing frequencies involved (10 - 12 act $/ \mathrm{min}$ ).

\subsubsection{Inspiration Time}

The application of the second Kirchhoff's law to the circuit of Figure 3 provides for the following equation:

$$
p_{A W i}\left(t_{i}\right)-R_{I N S} \phi_{I N S}\left(t_{i}\right)-p_{E A i}\left(t_{i}\right)=0
$$

AD_TRIANG (Figure 2) requires the following expression of $p_{A W i}\left(t_{i}\right)$ :

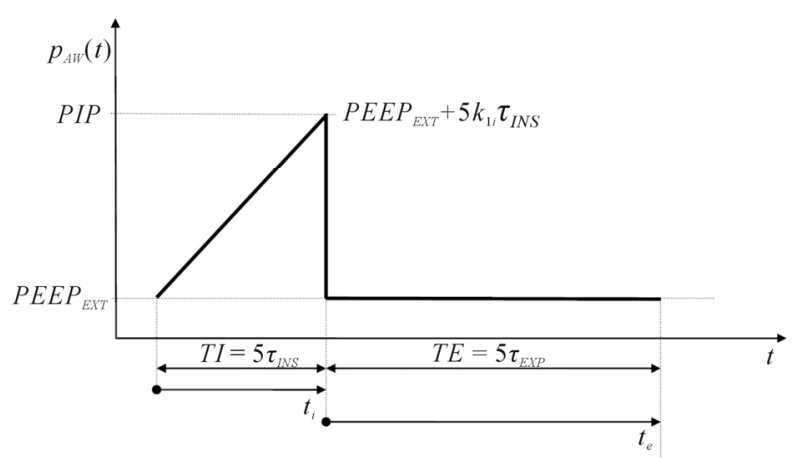

Figure 2. The advanced triangular waveform as airways pressure $\left(p_{A W}(t)\right)$ excitation (AD_TRIANG). 


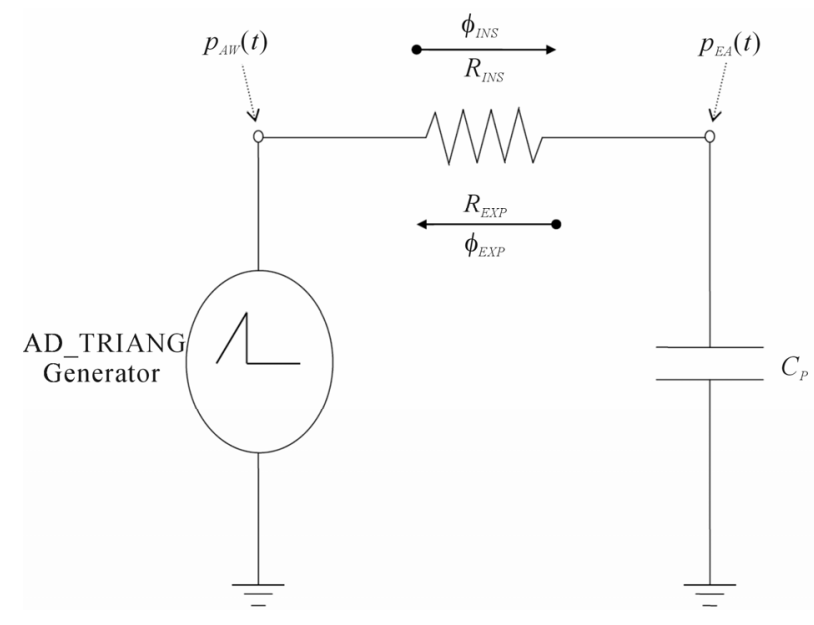

Figure 3. Electrical-equivalent circuit of AD_TRIANG generator connected to the patient's airways.

$$
p_{A W i}\left(t_{i}\right)=P E E P_{E X T}+k_{1 i} t_{i}
$$

where $k_{1 i}$ is the slope of $p_{A W_{i}}\left(t_{i}\right)$ linear increase with time $\left(t_{i}\right)$.

As it is well known, $\phi_{I N S}\left(t_{i}\right)$ is defined as the time derivation of $v_{P i}\left(t_{i}\right)$, as follows:

$$
\phi_{I N S}\left(t_{i}\right)=\frac{\mathrm{d} v_{P i}\left(t_{i}\right)}{\mathrm{d} t_{i}}
$$

Considering (3) as well as by inserting both (20) and (21) into (19), the following equation results:

$$
P E E P_{E X T}+k_{1 i} t_{i}-R_{I N S} \frac{\mathrm{d} v_{P i}\left(t_{i}\right)}{\mathrm{d} t_{i}}-\frac{v_{P i}\left(t_{i}\right)}{C_{P}}=0
$$

In order to solve Eq.22, i.e. to find out the transient and steady expressions of $v_{P i}\left(t_{i}\right)$, it is useful to transform it from time $\left(t_{i}\right)$ to Laplace $(s)$ variable domains, as follows:

$$
\frac{P E E P_{E X T}}{s}+\frac{k_{1 i}}{s^{2}}-R_{I N S}\left[s v_{P i}(s)-v_{P i}(0)\right]-\frac{v_{P i}(s)}{C_{P}}=0
$$

On account of both (1) and (9), the solution of (23) consists in the following expression:

$$
v_{P i}(s)=\frac{F R C s^{2}+\frac{F R C}{\tau_{I N S}} s+\frac{k_{1 i}}{R_{I N S}}}{s^{2}\left(s+\frac{1}{\tau_{I N S}}\right)}
$$

Eq.24 can be properly decomposed as follows:

$$
v_{P i}(s)=\frac{A}{s^{2}}+\frac{B}{s}+\frac{C}{s+\frac{1}{\tau_{I N S}}}
$$

The unknown constants $\mathrm{A}, \mathrm{B}$ and $\mathrm{C}$ can be determined by setting Eq.24 equal to Eq.25, resulting as follows:

$$
\begin{gathered}
A=k_{1 i} C_{P} \\
B=C_{P}\left(P E E P_{E X T}-k_{1 i} \tau_{I N S}\right)=F R C-k_{1 i} C_{P} \tau_{I N S} \\
C=k_{1 i} C_{P} \tau_{I N S}
\end{gathered}
$$

Finally, by inserting (26), (27) and (28) into (25), the following expression results:

$$
v_{P i}(s)=\frac{k_{1 i} C_{P}}{s^{2}}+\frac{F R C-k_{1 i} C_{P} \tau_{I N S}}{s}+\frac{k_{1 i} C_{P} \tau_{I N S}}{s+\frac{1}{\tau_{I N S}}}
$$

According to the Inverse Laplace Transform of (29), the function $v_{P i}\left(t_{i}\right)$ assumes the following expression:

$v_{P i}\left(t_{i}\right)=F R C+k_{1 i} C_{P}\left[t_{i}-\tau_{I N S}\left(1-\mathrm{e}^{-\frac{t_{i}}{\tau_{I N S}}}\right)\right]$

The functions $\phi_{I N S}\left(t_{i}\right)$ and $p_{E A i}\left(t_{i}\right)$ can be determined considering (21) and (3), respectively, as follows:

$$
\begin{gathered}
\phi_{I N S}\left(t_{i}\right)=k_{1 i} C_{P}\left(1-\mathrm{e}^{-\frac{t_{i}}{\tau_{I N S}}}\right) \\
p_{E A i}\left(t_{i}\right)=P E E P_{E X T}+k_{1 i}\left[t_{i}-\tau_{I N S}\left(1-\mathrm{e}^{-\frac{t_{i}}{\tau_{I N S}}}\right)\right]
\end{gathered}
$$

The functions $v_{P i}\left(t_{i}\right), \phi_{I N S}\left(t_{i}\right)$ and $p_{E A i}\left(t_{i}\right)$ are reported in Figure 4, Figure 5 and Figure 6, respectively.

The difference between $p_{A W i}\left(t_{i}\right)$ and $p_{E A i}\left(t_{i}\right)\left(\Delta p_{i}\left(t_{i}\right)\right)$ can be determined from both (20) and (32), as follows (Figure 6):

$$
\Delta p_{i}\left(t_{i}\right)=k_{1 i} \tau_{I N S}\left(1-\mathrm{e}^{-\frac{t_{i}}{\tau_{I N S}}}\right)
$$

The same result of (33) could also be obtained by inserting (31) into (19).

Considering (30), (31) and (32) at the beginning of inspiration time $\left(t_{i}=0\right)$, the following expressions result:

$$
\begin{aligned}
& v_{p i}(0)=F R C \\
& \phi_{I N S}(0)=0 \\
& p_{E A i}(0)=P E E P_{E X T}=p_{A W i}(0)
\end{aligned}
$$

(34) and (36) fit well (9) and (8), respectively (Figure 4 and Figure 6), according to the same steady conditions occurring in AD_SQUARE at the beginning of inspiration time $\left(t_{i}=0\right)$ and thus at the end of last expiration time $\left(t_{e}=T E\right)$.

If $T I$ is set equal to the time required for reaching the 


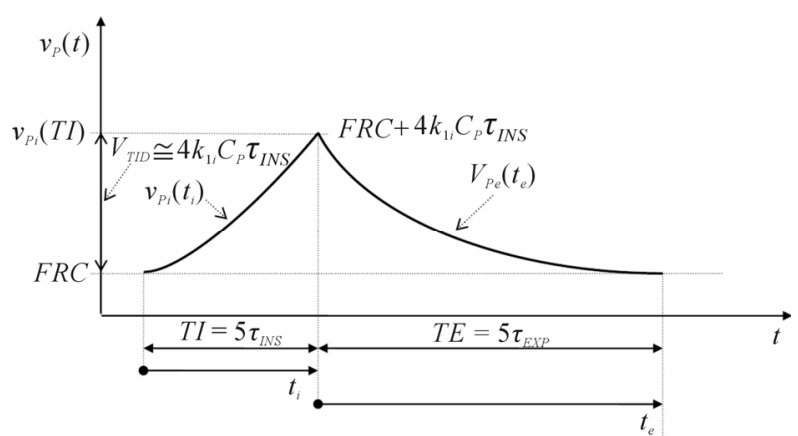

Figure 4. Lung volume $\left(v_{P}(t)\right)$ as a function of time $(t)$ resulting from the application of AD_TRIANG.

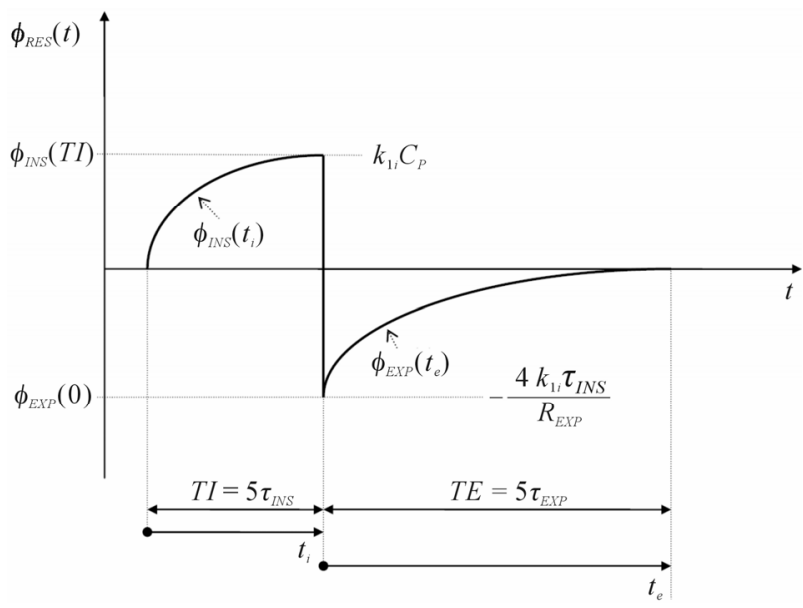

Figure 5. Respiratory airflow $\left(\phi_{R E S}(t)\right)$ as a function of time $(t)$ resulting from the application of AD_TRIANG.

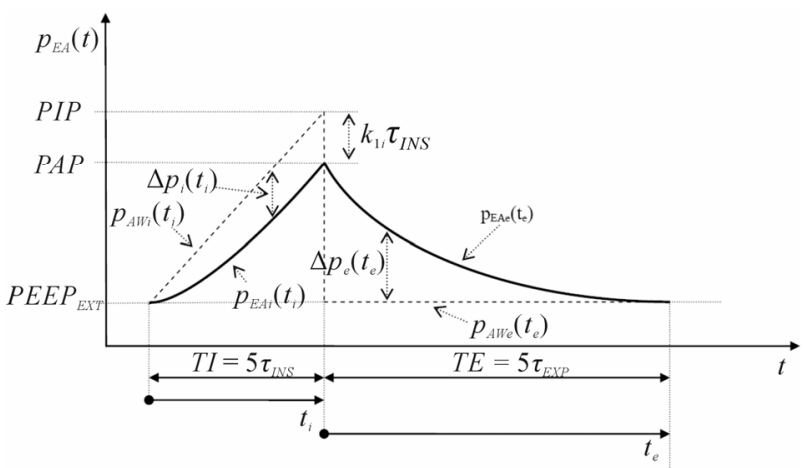

Figure 6. Endoalveolar pressure $\left(p_{E A}(t)\right)$ as a function of time $(t)$ resulting from the application of AD_TRIANG.

steady condition, i.e. the end of transient inspiration time $\left(t_{T I}=5 \tau_{I N S}\right),(20),(30),(31),(32)$ and (33) provide for the following expressions:

$$
\begin{aligned}
p_{A W i}(T I= & \left.5 \tau_{I N S}\right)=P I P=P E E P_{E X T}+5 k_{1 i} \tau_{I N S} \\
v_{p i}\left(T I=5 \tau_{I N S}\right) & =F R C+k_{1 i} C_{P}\left[5 \tau_{I N S}-\tau_{I N S}\left(1-\mathrm{e}^{-5}\right)\right] \\
& \cong F R C+4 k_{1 i} C_{P} \tau_{I N S}
\end{aligned}
$$

$$
\begin{aligned}
& \phi_{I N S}\left(T I=5 \tau_{I N S}\right)=k_{1 i} C_{P}\left(1-\mathrm{e}^{-5}\right) \cong k_{1 i} C_{P} \\
& p_{E A i}\left(T I=5 \tau_{I N S}\right)=P A P \cong P E E P_{E X T}+4 k_{1 i} \tau_{I N S} \\
& \Delta p_{i}\left(T I=5 \tau_{I N S}\right)=P I P-P A P \cong k_{1 i} \tau_{I N S}
\end{aligned}
$$

(39) establishes that at the end of transient time $\left(t_{i}=T I=\right.$ $\left.5 \tau_{I N S}\right)$, the increase of $\phi_{I N S}$ reaches a saturation value given by the product of $C_{P}$ with the linear slope $\left(k_{1 i}\right)$ selected for $p_{A W}$ (Figure 5). This result is remarkable since $\phi_{I N S}\left(5 \tau_{I N S}\right)$ being independent on $R_{I N S}$, provides for a more physiological $\phi_{I N S}$ waveform adapting itself to lung elastic characteristic $\left(C_{P}\right)$. Moreover, $\phi_{I N S}\left(5 \tau_{I N S}\right)$ can be adequately adjusted by $k_{1 i}$ regulation for a proper compensation of the actual $C_{P}$ value.

According to our purpose, (35) establishes the real smoothing of $\phi_{R E S}$ discontinuity occurring at the beginning of every inspiration time (Figure 5). Unfortunately, unlike AD_SQUARE, according to (39), the final value of $\phi_{I N S}\left(\phi_{I N S}\left(5 \tau_{I N S}\right)\right)$ is different from zero (Figure 5$)$. Such problem will be soon after removed with $\mathrm{AD}$ TRAPEZ (§ 3.4).

Both (33) and (41) establish that $\Delta p_{i}$ increases from zero to a saturation value (Figure 6) given by the product $k_{1 i} \tau_{I N S}$ reached at the end of transient time $\left(t_{i}=T I=\right.$ $\left.5 \tau_{I N S}\right)$. Moreover, (32) establishes that during the transient time $\left(0 \leq t_{i} \leq T I\right)$ the second time derivation of $p_{E A i}\left(t_{i}\right)$ is positive, the time rate of $p_{E A i}\left(t_{i}\right)$ increasing from 0 to $k_{1 i}$, that is the slope selected for $p_{A W_{i}}\left(t_{i}\right)$. The last results are equally remarkable if compared to those obtained with AD_SQUARE. Unlike AD_SQUARE, indeed, where $p_{E A i}\left(t_{i}\right)$ waveform can be controlled only by selecting the maximum $(P I)$ constant level of $p_{A W}$ (control of first order), in AD_TRIANG $p_{E A i}\left(t_{i}\right)$ waveform can be much safely controlled by selecting $k_{1 i}$ value under of which the $p_{E A i}\left(t_{i}\right)$ increasing rate with time is certainly kept (control of second order).

Considering both (34) and (38), $V_{\text {TID }}$ for an inspiration time equal to $T I\left(V_{T I D}(T I)\right)$, results as follows:

$$
V_{T I D}(T I)=v_{p i}\left(T I=5 \tau_{I N S}\right)-v_{p i}(0) \cong 4 k_{1 i} C_{P} \tau_{I N S}
$$

From both (12) and (42), $V_{M I N}$ for an inspiration time equal to $T I\left(V_{M I N}(T I)\right)$, results as follows:

$$
V_{\text {MIN }}(T I) \cong \frac{48 k_{1 i} \tau_{I N S}}{R_{I N S}+R_{E X P}}
$$

(43) takes into account that $T E$ is set equal to $5 \tau_{E X P}(\S$ 3.3.2).

Expressions (37)-(43) provide the rationale for the optimization of ventilation control in AD_TRIANG during the time of inspiration.

$k_{1 \mathrm{i}}$ value required for delivering in the same time $(T I=$ $\left.5 \tau_{I N S}\right)$ the same $V_{T I D}$ as in AD_SQUARE $\left(k_{\text {squii }}\right)$ can be determined by setting (42) equal to (11), obtaining the 
following expression:

$$
k_{\text {squ1i }} \cong \frac{P I-P E E P_{E X T}}{4 \tau_{I N S}}
$$

Under the last condition $\left(k_{1 i}=k_{\text {squ1i }}\right)$, the following expressions result:

$$
\begin{gathered}
P I P \cong P I+\frac{P I-P E E P_{E X T}}{4} \\
P A P \cong P I \\
P I P-P A P \cong \frac{P I-P E E P_{E X T}}{4} \\
\phi_{I N S}(T I)=\frac{P I P-P A P}{R_{I N S}} \cong \frac{P I-P E E P_{E X T}}{4 R_{I N S}}
\end{gathered}
$$

So, the peak of $\phi_{I N S}$ and PIP required in AD_TRIANG for delivering in the same time $\left(T I=5 \tau_{I N S}\right)$ the same $V_{T I D}$ and thus for reaching the same $P A P$ as in AD_SQUARE shows a $75 \%$ reduction and increases a quarter of the difference between upper $(P I)$ and lower $\left(P E E P_{E X T}\right) p_{A W}$ constant levels, respectively.

The diagnostic procedure has to be implemented as follows. According to both (1) and (31), the measurement of the time required for reaching the end of transient inspiration time $\left(t_{T I}=5 \tau_{I N S}\right)$, i.e. for observing a differential increase of $\phi_{\text {INS }}$ with time lower than one percent $(1 \%)$ or saturated $\phi_{I N S}\left(\phi_{I N S}\left(t_{T I}=5 \tau_{I N S}\right)\right)$, is useful for the determination of $\tau_{\text {INS }}$, as follows:

$$
\tau_{\text {INS }} \cong \frac{t_{T I}}{5}
$$

According to (39), for a given $k_{1 i}$ value, the monitoring of $\phi_{I N S}\left(t_{T I}=5 \tau_{I N S}\right)$ leads to the determination of $C_{P}$, as follows:

$$
C_{P} \cong \frac{\phi_{I N S}\left(t_{T I}=5 \tau_{I N S}\right)}{k_{1 i}}
$$

From (1), (49) and (50), $R_{I N S}$ can be determined as follows:

$$
R_{I N S}=\frac{\tau_{I N S}}{C_{P}} \cong \frac{t_{T I} k_{1 i}}{5 \phi_{I N S}\left(t_{T I}=5 \tau_{I N S}\right)}
$$

Once $\tau_{I N S}, C_{P}$ and $R_{I N S}$ have been determined, TI should be set equal to the measured $t_{T I}\left(T I=t_{T I}\right)$ and $k_{1 i}$ regulation should be performed in order to fit the clinical requirements on PIP, PAP, $V_{T I D}$ or $V_{M I N}$ through (37), (40), (42) or (43), respectively. In particular, concerning with dual-control mode, $k_{1 i}$ values ensuring the pre-set $V_{T I D}$ $\left(k_{1 i T I D}\right)$ or $V_{M I N}\left(k_{1 i M I N}\right)$ can be determined from $(42)$ or (43), as follows:

$$
k_{1 i T I D} \cong \frac{V_{T I D}}{4 C_{P} \tau_{I N S}}
$$

$$
k_{1 i M I N} \cong \frac{V_{M I N}\left(R_{I N S}+R_{E X P}\right)}{48 \tau_{I N S}}
$$

$P I P$ values resulting from $V_{T I D}\left(P I P_{T I D}\right)$ or $V_{M I N}\left(P I P_{M I N}\right)$ dual-control mode, can be obtained by inserting (52) or (53) into (37), as follows:

$$
\begin{gathered}
P I P_{T I D} \cong P E E P_{E X T}+\frac{5 V_{T I D}}{4 C_{P}} \\
P I P_{M I N} \cong P E E P_{E X T}+\frac{5 V_{M I N}\left(R_{I N S}+R_{E X P}\right)}{48}
\end{gathered}
$$

(54) and (55) show that PIP TID or PIP ${ }_{M I N}$ are independent on $R_{R E S}$ or $C_{P}$, respectively. That is extremely relevant from both physiopathological and clinical point of view since an increase of $R_{R E S}$ (obstructive process) or a reduction of $C_{P}$ (restrictive process) does not affect the maximum $p_{A W}$ value reached for dual-control mode with pre-set $V_{T I D}$ or $V_{M I N}$, respectively.

The compensation procedure is required in order to assimilate ALVS with an ideal $p_{A W}$ generator providing for a real AD_TRIANG, i.e. triangular waveform as $p_{A W}$ excitation insensitive to patient's respiratory characteristics and ventilator settings, through a proper triangular waveform of external resistance $\left(R_{E X T}\right)$ of ALVS which controls $p_{A W}$ [9]. The electrical-equivalent network of ALVS is shown in Figure 7. According to ALVS configuration and performances [9], the compensation procedure requires that during both inspiration and expiration the airflow crossing $R_{E X T}$, i.e. the external airflow $\left(\phi_{E X T}\right)$, must be kept constant and equal to the equilibrium value assumed in initial steady conditions during apnea $\left(\phi_{E X T 0}\right)$, for which the following expressions result:

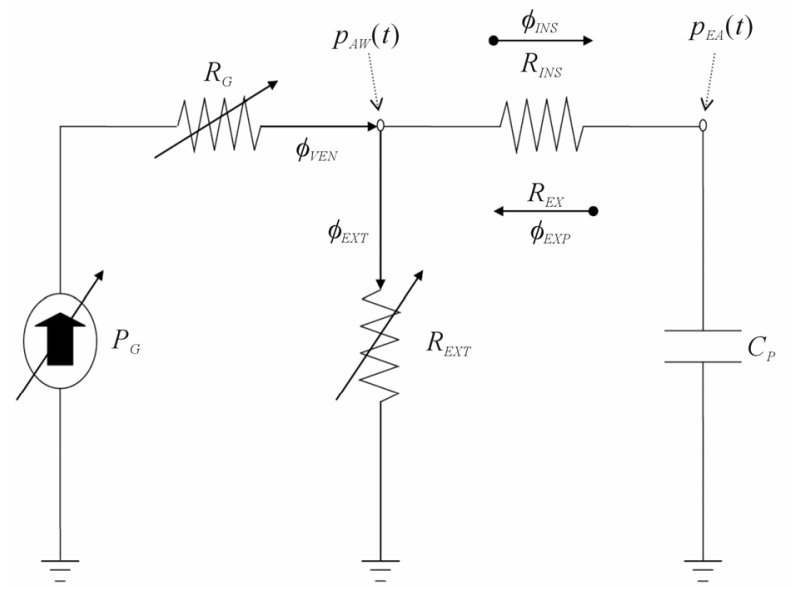

Figure 7. Electrical-equivalent network of ALVS. The components crossed with folded arrows are devices whose characteristic parameter output can be varied according to input setting control. 


$$
\begin{aligned}
& \phi_{R E S} \equiv 0 \\
& \phi_{E X T 0} \equiv \phi_{V E N 0}=\frac{P_{G}}{R_{G 0}} \\
& P E E P_{E X T}=\phi_{E X T 0} R_{E X T 0}=\frac{P_{G} R_{E X T 0}}{R_{G 0}}
\end{aligned}
$$

$\phi_{V E N 0}$ is the steady equilibrium value assumed by the ventilation airflow $\left(\phi_{V E N}\right)$, i.e. the airflow delivered by the generator. $R_{E X T 0}$ is the lowest $R_{E X T}$ value set for $P E E P_{E X T}$ regulation by means of (58). $P_{G}$ and $R_{G 0}$ are the output pressure and equilibrium value assumed by the internal resistance $\left(R_{G}\right)$ of ALVS generator, respectively, both set for $\phi_{V E N O}$ regulation by means of (57), according to the following initial steady condition:

$$
R_{G 0} \gg R_{E X T 0}
$$

Considering (58), (59) implies the following condition:

$$
P_{G} \gg P E E P_{E X T}
$$

If $\phi_{E X T}$ is stabilized on constant steady $\phi_{E X T 0}$ value, AD_TRIANG can be obtained by modeling $R_{E X T}$ waveform $\left(R_{E X T}(t)\right)$ as linear increasing of $R_{E X T}$ from $R_{E X T 0}$ to its maximum value ( $\left.R_{E X T}^{*}\right)$ during inspiration $\left(0 \leq t_{i} \leq T I\right)$ and as instantaneous fall of $R_{E X T}$ from $R_{E X T}^{*}$ to $R_{E X T 0}$ followed by constant keeping of $R_{E X T}$ on $R_{E X T 0}$ during expiration $\left(0 \leq t_{e} \leq T E\right)$. From (57) and (58), $R_{E X T}^{*}$ is determined as follows:

$$
R_{E X T}^{*}=\frac{P I P}{\phi_{E X T 0}}=\frac{R_{G 0} P I P}{P_{G}}=\frac{R_{E X T 0} P I P}{P E E P_{E X T}}
$$

In particular, concerning with dual-control mode, $R_{E X T}^{*}$ values ensuring the pre-set $V_{T I D}\left(R_{E X T}^{*}\right)$ or $V_{M I N}$ $\left(R_{E X T}^{* M I N}\right)$ can be obtained by inserting (54) or (55) into (61), respectively, and considering (9), as follows:

$$
\begin{gathered}
R_{E X T}^{* T I D} \cong R_{E X T 0}\left(1+\frac{5 V_{T I D}}{4 F R C}\right) \\
R_{E X T}^{* M I N} \cong R_{E X T 0}\left[1+\frac{5 V_{M I N}\left(R_{I N S}+R_{E X P}\right)}{48 P E E P_{E X T}}\right]
\end{gathered}
$$

So, $R_{E X T}\left(t_{i}\right)$ to be implemented for dual-control mode during inspiration $\left(0 \leq t_{i} \leq T I\right)$ with pre-set $V_{T I D}$ $\left(R_{E X T}\left(t_{i}\right)^{T I D}\right)$ or $V_{M I N}\left(R_{E X T}\left(t_{i}\right)^{M I N}\right)$, assumes the following expressions:

$$
\begin{aligned}
R_{E X T}\left(t_{i}\right)^{T I D} \cong R_{E X T 0}\left(1+\frac{V_{T I D} t_{i}}{4 F R C \tau_{I N S}}\right) \\
R_{E X T}\left(t_{i}\right)^{M I N} \cong R_{E X T 0}\left[1+\frac{V_{M I N}\left(R_{I N S}+R_{E X P}\right) t_{i}}{48 R_{I N S} F R C}\right]
\end{aligned}
$$

The stabilization of $\phi_{E X T}$ during inspiration $\left(\phi_{E X T i}\left(t_{i}\right)\right)$ on $\phi_{\text {EXTO }}$ can be carried out by proper modeling $\phi_{V E N}$ waveform during inspiration $\left(\phi_{V E N i}\left(t_{i}\right)\right)$ according to the first
Kirchhoff's low applied at airways node of ALVS's network (Figure 7):

$$
\phi_{V E N i}\left(t_{i}\right)=\phi_{E X T i}\left(t_{i}\right)+\phi_{I N S}\left(t_{i}\right)=\phi_{E X T 0}+\phi_{I N S}\left(t_{i}\right)
$$

By inserting both (57) and (31) into (66), the following expression results:

$$
\phi_{V E N i}\left(t_{i}\right)=\frac{P_{G}}{R_{G 0}}+k_{1 i} C_{P}\left(1-\mathrm{e}^{-\frac{t_{i}}{\tau_{I N S}}}\right)
$$

The second Kirchhoff's low applied to the circuit of ALVS generator (Figure 7), assumes the following expression:

$$
P_{G}-R_{G i}\left(t_{i}\right) \phi_{V E N i}\left(t_{i}\right)-p_{A W i}\left(t_{i}\right)=0
$$

$R_{G i}\left(t_{i}\right)$ is the $R_{G}$ waveform to be implemented for performing an effective compensation procedure during inspiration. By inserting both (67) and (20) into (68), $R_{G i}\left(t_{i}\right)$ can be determined as follows:

$$
R_{G i}\left(t_{i}\right)=\frac{P_{G}-P E E P_{E X T}-k_{1 i} t_{i}}{\frac{P_{G}}{R_{G 0}}+k_{1 i} C_{P}\left(1-\mathrm{e}^{-\frac{t_{i}}{\tau_{I N S}}}\right)}
$$

From (69), on account of (60), the maximum $\left(R_{G i}{ }^{*}\right)$ and minimum $\left(R_{G i}^{\prime}\right)$ values assumed by $R_{G i}\left(t_{i}\right)$ at the beginning $\left(t_{i}=0\right)$ and at end $\left(t_{i}=T I\right)$ of the time during inspiration $\left(t_{i}\right)$, respectively, result as follows:

$$
\begin{aligned}
& R_{G i}^{*}=R_{G i}(0)=R_{G 0} \\
& R_{G i}^{\prime}=R_{G i}(T I) \cong \frac{P_{G}-P I P}{\frac{P_{G}}{R_{G 0}}+k_{1 i} C_{P}}
\end{aligned}
$$

So, considering that in coincidence with the end of inspiration $\left(t_{i}=T I\right)$, i.e. the end of transient inspiration time, $R_{G i}$ and $R_{E X T i}$ assume their minimum $\left(R_{G i}^{\prime}\right)$ and maximum $\left(R_{E X T}^{*}\right)$ values, respectively, $R_{G i}$ modeling must take into account the following final steady condition:

$$
R_{G i}^{\prime} \gg R_{E X T}^{*}
$$

According to both (70) and (71), i.e. to condition $R_{G i}^{\prime}$ $<R_{G 0}$ and considering (61), (72) implies the following condition:

$$
P_{G} \gg P I P
$$

Obviously, (72) and (73) replace (59) and (60), respectively. On account of both (73) and (58), (69) and (71) reduce to the following expressions, respectively:

$$
\begin{aligned}
R_{G i}\left(t_{i}\right) \cong & \frac{P_{G}}{\frac{P_{G}}{R_{G 0}}+k_{1 i} C_{P}\left(1-\mathrm{e}^{-\frac{t_{i}}{\tau_{I N S}}}\right)} \\
= & \frac{R_{G 0} P E E P_{E X T}}{P E E P_{E X T}+k_{1 i} R_{E X T 0} C_{P}\left(1-\mathrm{e}^{-\frac{t_{i}}{\tau_{I N S}}}\right)}
\end{aligned}
$$




$$
R_{G i}^{\prime}=R_{G i}(T I) \cong \frac{P_{G}}{\frac{P_{G}}{R_{G 0}}+k_{1 i} C_{P}}=\frac{R_{G 0} P E E P_{E X T}}{P E E P_{E X T}+k_{1 i} R_{E X T 0} C_{P}}
$$

Moreover, in order to avoid that $R_{G i}^{\prime}$ reaches unpractical reduced values, the following condition should be properly taken into account:

$$
R_{G i}^{\prime} \geq \frac{R_{G 0}}{2}
$$

Considering (75), (76) leads to the following functional limitation on $k_{1 i}$ value:

$$
k_{1 i} \leq \frac{P_{G}}{C_{P} R_{G 0}}=\frac{P E E P_{E X T}}{C_{P} R_{E X T 0}}
$$

The function $R_{G i}\left(t_{i}\right)$ is reported in Figure 8 .

\subsubsection{Expiration time}

The application of the second Kirchhoff' law to the circuit of Figure 3 provides for the following equation:

$$
p_{\text {AWe }}\left(t_{e}\right)+R_{E X P} \phi_{E X P}\left(t_{e}\right)-p_{E A e}\left(t_{e}\right)=0
$$

As AD_SQUARE, AD_TRIANG requires the following expression of $p_{\text {AWe }}\left(t_{e}\right)$ :

$$
p_{\text {AWe }}\left(t_{e}\right) \equiv P E E P_{E X T}
$$

As it is well known, $\phi_{E X P}\left(t_{e}\right)$ is defined as the time derivation of $v_{P e}\left(t_{e}\right)$, as follows:

$$
\phi_{E X P}\left(t_{e}\right)=-\frac{\mathrm{d} v_{P e}\left(t_{e}\right)}{\mathrm{d} t_{e}}
$$

Considering (3) as well as by inserting both (79) and (80) into (78), the following equation results:

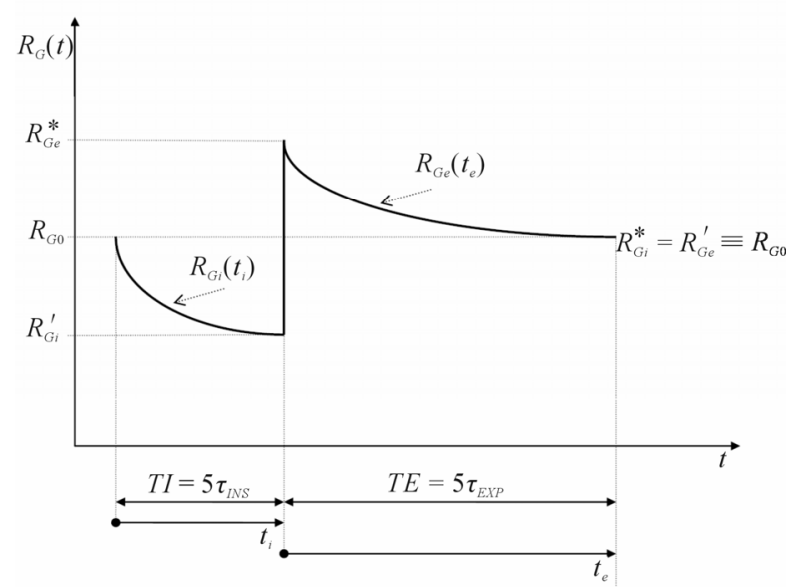

Figure 8. Internal resistance $\left(R_{G}(t)\right)$ of ALVS generator as a function of time $(t)$ to be implemented for performing the compensation procedure in AD_TRIANG.

$$
P E E P_{E X T}-R_{E X P} \frac{\mathrm{d} v_{P e}\left(t_{e}\right)}{\mathrm{d} t_{e}}-\frac{v_{P e}\left(t_{e}\right)}{C_{P}}=0
$$

In order to solve Eq.81, i.e. to find out the transient and steady expressions of $v_{P e}\left(t_{e}\right)$, it is useful to transform it from time $\left(t_{e}\right)$ to Laplace (s) variable domains, as follows:

$$
\frac{P E E P_{E X T}}{s}-R_{E X P}\left[s v_{P e}(s)-v_{P e}(0)\right]-\frac{v_{P e}(s)}{C_{P}}=0
$$

From both (2) and (38), on account of continuity condition on $v_{P}$ when the switching between inspiration and expiration takes place $\left(v_{P e}(0) \equiv v_{P i}(T I)\right)$, the solution of (82) consists in the following expression:

$$
v_{P e}(s)=\frac{\left(F R C+4 k_{1 i} C_{P} \tau_{I N S}\right) s+\frac{F R C}{\tau_{E X P}}}{s\left(s+\frac{1}{\tau_{E X P}}\right)}
$$

Eq.83 can be properly decomposed as follows:

$$
v_{P e}(s)=\frac{A}{s}+\frac{B}{s+\frac{1}{\tau_{E X P}}}
$$

The unknown constants A and B can be determined by setting Eq.83 equal to Eq.84, resulting as follows:

$$
\begin{aligned}
& A=F R C \\
& B=4 k_{1 i} C_{P} \tau_{I N S}
\end{aligned}
$$

Finally, by inserting (85) and (86) into (84), the following expression results:

$$
v_{P e}(s)=\frac{F R C}{s}+\frac{4 k_{1 i} C_{P} \tau_{I N S}}{s+\frac{1}{\tau_{E X P}}}
$$

According to the Inverse Laplace Transform of (87), the function $v_{P e}\left(t_{e}\right)$ assumes the following expression:

$$
v_{P e}\left(t_{e}\right)=F R C+4 k_{1 i} C_{P} \tau_{I N S} \mathrm{e}^{-\frac{t_{e}}{\tau_{E X P}}}
$$

The functions $\phi_{E X P}\left(t_{e}\right)$ and $p_{E A e}\left(t_{e}\right)$ can be determined considering (80) and (3), respectively, as follows:

$$
\begin{gathered}
\phi_{E X P}\left(t_{e}\right)=\frac{4 k_{1 i} \tau_{I N S}}{R_{E X P}} \mathrm{e}^{-\frac{t_{e}}{\tau_{E X P}}} \\
p_{E A e}\left(t_{e}\right)=P E E P_{E X T}+4 k_{1 i} \tau_{I N S} \mathrm{e}^{-\frac{t_{e}}{\tau_{E X P}}}
\end{gathered}
$$

The functions $v_{P e}\left(t_{e}\right), \phi_{E X P}\left(t_{e}\right)$ and $p_{E A e}\left(t_{e}\right)$ are reported in Figure 4, Figure 5 and Figure 6, respectively.

The difference between $p_{\text {AWe }}\left(t_{e}\right)$ and $p_{E A e}\left(t_{e}\right)\left(\Delta p_{e}\left(t_{e}\right)\right)$ can be determined from both (79) and (90), as follows 
(Figure 6):

$$
\Delta p_{e}\left(t_{e}\right)=-4 k_{1 i} \tau_{I N S} v^{-\frac{t_{e}}{\tau_{E X P}}}
$$

The same result of (91) could also be obtained by inserting (89) into (78).

Considering (88), (89) and (90) at the beginning of expiration time $\left(t_{e}=0\right)$, the following expressions result:

$$
\begin{gathered}
v_{P e}(0)=F R C+4 k_{1 i} C_{P} \tau_{I N S}=v_{P i}(T I) \\
\phi_{E X P}(0)=\frac{4 k_{1 i} \tau_{I N S}}{R_{E X P}} \\
p_{E A e}(0)=P E E P_{E X T}+4 k_{1 i} \tau_{I N S}=p_{E A i}(T I
\end{gathered}
$$

(92) and (94) fit well (38) and (40), respectively, according to continuity condition required between the end of inspiration and the beginning of expiration (Figure 4 and Figure 6). Unlike our purpose, (39) together with (93) establish a considerable discontinuity occurring on $\phi_{R E S}$ at the end of every inspiration when the switching between inspiration and expiration takes place (Figure 5). Such problem will be soon after removed with AD TRAPEZ (§ 3.4).

If $T E$ is set equal to the time required for reaching the steady condition, i.e. the end of transient expiration time $\left(t_{T E}=5 \tau_{E X P}\right)$, (88), (89), (90) and (91) provide for the following expressions:

$$
\begin{aligned}
& v_{P e}\left(T E=5 \tau_{I N S}\right)=F R C+4 k_{1 i} C_{P} \tau_{I N S} \mathrm{e}^{-5} \cong F R C=v_{P i}(0) \\
& \phi_{E X P}\left(T E=5 \tau_{I N S}\right)=\frac{\left(4 k_{1 i} \tau_{I N S}\right)}{R_{E X P}} \mathrm{e}^{-5} \cong 0 \\
& p_{E A e}\left(T E=5 \tau_{I N S}\right)=P E E P_{E X T}+4 k_{1 i} \tau_{I N S} \mathrm{e}^{-5} \\
& \cong P E E P_{E X T}=p_{E A i}(0) \\
& \Delta p_{e}\left(T E=5 \tau_{I N S}\right)=-4 k_{1 i} \tau_{I N S} \mathrm{e}^{-5} \cong 0
\end{aligned}
$$

(95) and (97) fit well (34) and (36), respectively, according to continuity condition required at the transition between the end of every expiration and the beginning of the following inspiration (Figure 4 and Figure 6). Moreover, according to our purpose, (96) together with (35) establish the real elimination of $\phi_{R E S}$ discontinuity occurring in coincidence with such a transition (Figure 5).

The diagnostic procedure has been implemented as follows. According to both (2) and (89), the measurement of the time required for reaching the end of transient expiration time $\left(t_{T E}=5 \tau_{E X P}\right)$, i.e. for observing a ninety nine per cent $(99 \%)$ reduction of $\phi_{E X P}$ with regard to its initial value $\left(\phi_{E X P}(0)\right)$, is useful for the determination of $\tau_{E X P}$, as follows:

$$
\tau_{E X P} \cong \frac{t_{T E}}{5}
$$

According to (93), for a given $k_{1 i}$ and $\tau_{I N S}$ values, the monitoring of $\phi_{E X P}(0)$ leads to the determination of $R_{E X P}$, as follows:

$$
R_{E X P}=\frac{4 k_{1 i} \tau_{I N S}}{\phi_{E X P}(0)}
$$

From (2), (99) and (100), $C_{P}$ can be determined as follows:

$$
C_{P}=\frac{\tau_{E X P}}{R_{E X P}} \cong \frac{t_{T E} \phi_{E X P}(0)}{20 k_{1 i} \tau_{I N S}}
$$

(101) can be employed for confirming the result obtained with (50). Once $\tau_{E X P}, R_{E X P}$ and $C_{P}$ have been determined, $T E$ should be set equal to the measured $t_{T E}$ (TE $\left.=t_{T E}\right)$.

So, considering (58) and (79), $R_{E X T}\left(t_{e}\right)$ to be implemented for dual-control mode during expiration $\left(0 \leq t_{e} \leq\right.$ $T E)$ with pre-set $V_{T I D}\left(R_{E X T}\left(t_{e}\right)^{T I D}\right)$ or $V_{M I N}\left(R_{E X T}\left(t_{e}\right)^{M I N}\right)$, assume the following expression:

$$
R_{E X T}\left(t_{e}\right)^{T I D}=R_{E X T}\left(t_{e}\right)^{M I N} \equiv R_{E X T 0}
$$

The compensation procedure, i.e. the stabilization of $\phi_{E X T}$ during expiration $\left(\phi_{\text {EXTe }}\left(t_{e}\right)\right)$ on $\phi_{\text {EXT0 }}$ can be carried out by proper modeling $\phi_{V E N}$ waveform during expiration $\left(\phi_{V E N e}\left(t_{e}\right)\right)$ according to the first Kirchhoff's low applied at airways node of ALVS's network (Figure 7):

$$
\phi_{V E N e}\left(t_{e}\right)=\phi_{\text {EXTe }}\left(t_{e}\right)-\phi_{E X P}\left(t_{e}\right)=\phi_{E X T 0}-\phi_{E X P}\left(t_{e}\right)
$$

By inserting both (57) and (89) into (103), the following expression results:

$$
\phi_{V E N e}\left(t_{e}\right)=\frac{P_{G}}{R_{G 0}}-\frac{4 k_{1 i} \tau_{I N S}}{R_{E X P}} \mathrm{e}^{-\frac{t_{e}}{\tau_{E X P}}}
$$

The second Kirchhoff's low applied to the circuit of ALVS generator (Figure 7), assumes the following expression:

$$
P_{G}-R_{G e}\left(t_{e}\right) \phi_{V E N e}\left(t_{e}\right)-p_{A W e}\left(t_{e}\right)=0
$$

$R_{G e}\left(t_{e}\right)$ is the $R_{G}$ waveform to be implemented for performing an effective compensation procedure during expiration. By inserting both (104) and (79) into (105), $R_{G e}\left(t_{e}\right)$ can be determined as follows:

$$
R_{G e}\left(t_{e}\right)=\frac{P_{G}-P E E P_{E X T}}{\frac{P_{G}}{R_{G 0}}-\frac{4 k_{1 i} \tau_{I N S}}{R_{E X P}} \mathrm{e}^{-\frac{t_{e}}{\tau_{E X P}}}}
$$

From (106), on account of (60), the maximum $\left(R^{*}\right)$ and minimum $\left(R_{G e}^{\prime}\right)$ values assumed by $R_{G e}\left(t_{e}\right)$ at the beginning $\left(t_{e}=0\right)$ and at end $\left(t_{e}=T E\right)$ of the time during 
expiration $\left(t_{e}\right)$, respectively, result as follows:

$$
\begin{aligned}
& R_{G e}^{*}=R_{G e}(0) \cong \frac{P_{G}}{\frac{P_{G}}{R_{G 0}}-\frac{4 k_{1 i} \tau_{I N S}}{R_{E X P}}} \\
& R_{G e}^{\prime}=R_{G e}(T E) \cong R_{G 0}
\end{aligned}
$$

On account of both (58) and (60), (106) and (107) reduce to the following expressions, respectively:

$$
\begin{aligned}
& R_{G e}\left(t_{e}\right) \cong \frac{P_{G}}{\frac{P_{G}}{R_{G 0}}-\frac{4 k_{1 i} \tau_{I N S}}{R_{E X P}} \mathrm{e}^{-\frac{t_{e}}{\tau_{E X P}}}} \\
&= \frac{R_{G 0} P E E P_{E X T}}{P E E P_{E X T}-\frac{4 k_{1 i} R_{E X T 0} \tau_{I N S}}{R_{E X P}} \mathrm{e}^{-\frac{t_{e}}{\tau_{E X P}}}} \\
& R_{G e}^{*}=R_{G e}(0) \cong \frac{R_{G 0} P E E P_{E X T}}{P E E P_{E X T}-\frac{4 k_{11} R_{E X T 0} \tau_{I N S}}{R_{E X P}}}
\end{aligned}
$$

The function $R_{G e}\left(t_{e}\right)$ is reported in Figure 8.

So that, in conclusion, the implementation of (74) and (109) during the inspiration $\left(0 \leq t_{i} \leq T I\right)$ and expiration $\left(0 \leq t_{e} \leq T E\right)$ times, respectively, ensures the compensation procedure to be carried out, providing for an effective AD TRIANG.

From Figure 2, Figure 6, (12), (15), (16), (20), (32), (44), (79) and (90), it is easy to demonstrate that MAP and $M E P$ values of AD_TRIANG $\left(M A P_{t r i}\right.$ and $\left.M E P_{t r i}\right)$ result as follows:

$$
\begin{aligned}
M A P_{t r i} & =P E E P_{E X T}+\frac{\left(P I-P E E P_{E X T}\right) 0.625 \tau_{I N S}}{\left(\tau_{I N S}+\tau_{E X P}\right)} \\
M E P_{t r i} & \cong P E E P_{E X T} \\
+ & \frac{\left(P I-P E E P_{E X T}\right)\left(0.425 \tau_{I N S}+0.2 \tau_{E X P}\right)}{\left(\tau_{I N S}+\tau_{E X P}\right)}
\end{aligned}
$$

In comparison with (17), (111) shows a $37.5 \%$ reduction of the component of $M A P_{t r i}$ above PEEP $E X T$ with regard to the same component of $M A P_{s q u}$. Moreover, in comparison with (18), (112) shows a $31.3 \%$ reduction of the component of $M E P_{t r i}$ above $P E E P_{E X T}$ with regard to the same component of $M E P_{\text {squ }}$, if the ratio between $\tau_{E X P}$ and $\tau_{I N S}$ is estimated twice.

\subsection{Advanced Trapezoidal Waveform as Airways Pressure Excitation (AD_TRAPEZ)}

Figure 9 shows the advanced trapezoidal waveform as $p_{A W}$ excitation (AD_TRAPEZ). In AD_TRAPEZ the time of inspiration $\left(\overline{0} \leq t_{i} \leq T I\right)$ is divided into two sub

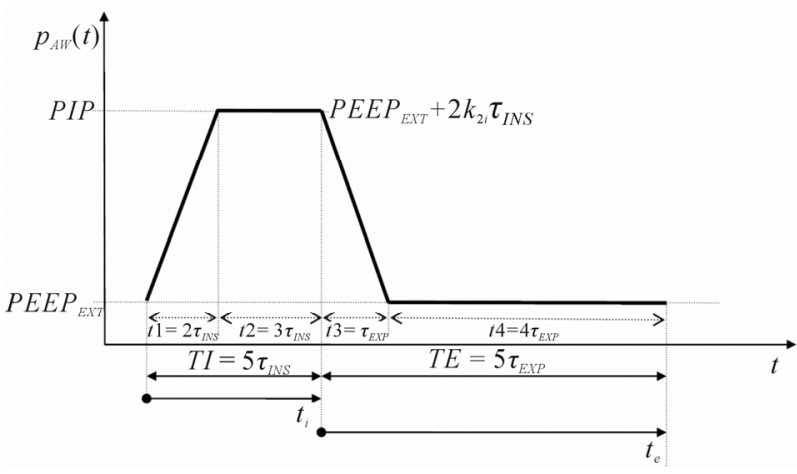

Figure 9. The advanced trapezoidal waveform as airways pressure $\left(p_{A W}(t)\right)$ excitation (AD_TRAPEZ).

sequent intervals of time lasting $t 1$ and $t 2$. During first $(0$ $\left.\leq t_{i} \leq t 1\right)$ and second $\left(t 1 \leq t_{i} \leq t 1+t 2=T I\right)$ intervals, $p_{A W}$ increases linearly with an higher slope $\left(k_{2 i}\right)$ from minimum or $P E E P_{E X T}$ to maximum or peak (PIP) values and keeps constant on PIP value, respectively. Therefore, $t 1$, $t 2$ and $k_{2 i}$ fit the following conditions:

$$
\begin{aligned}
& t 1+t 2=T I \\
& k_{2 i}>k_{1 i}
\end{aligned}
$$

As in AD_TRIANG, the linear increase of $p_{A W}$ has been selected for smoothing $\phi_{R E S}$ discontinuity occurring at the beginning of every inspiration, but its duration has been reduced $(t 1<T I)$ for having a following interval $(t 2$ $=T I-t 1)$ with constant $p_{A W}$ available for reducing to zero the final value of $\phi_{I N S}$. Unlike AD_SQUARE and AD_TRIANG, during the time of expiration $\left(0 \leq t_{e} \leq T E\right)$ $p_{A W}$ is no more kept constant on PEEP $P_{E X T}$. During the first interval of expiration $\left(0 \leq t_{e} \leq t 3\right), p_{A W}$ linearly falls with a slope $k_{2 e}$ from PIP to $P E E P_{E X T}$ values for reducing to zero the initial value of $\phi_{E X P}$, while during the second interval of expiration $\left(t 3 \leq t_{e} \leq t 3+t 4=T E\right) p_{A W}$ keeps constant on $P E E P_{E X T}$ value for reducing to zero the final value of $\phi_{E X P}$. Obviously, $t 3$ and $t 4$ fit the following condition:

$$
t 3+t 4=T E
$$

In such a way, according to our purpose, the discontinuity on $\phi_{R E S}$ occurring in AD TRIANG at the end of every inspiration when the switching between inspiration and expiration takes place can be completely removed.

AD_TRAPEZ can be carried out by connecting the patient's airways with an ideal generator creating a trapezoidal waveform of $p_{A W}$. The electrical-equivalent circuit of AD TRIANG generator connected to the patient's airways is shown in Figure 10.

\subsubsection{Inspiration Time}

Concerning the first interval of inspiration $\left(0 \leq t_{i} \leq t 1\right)$, 


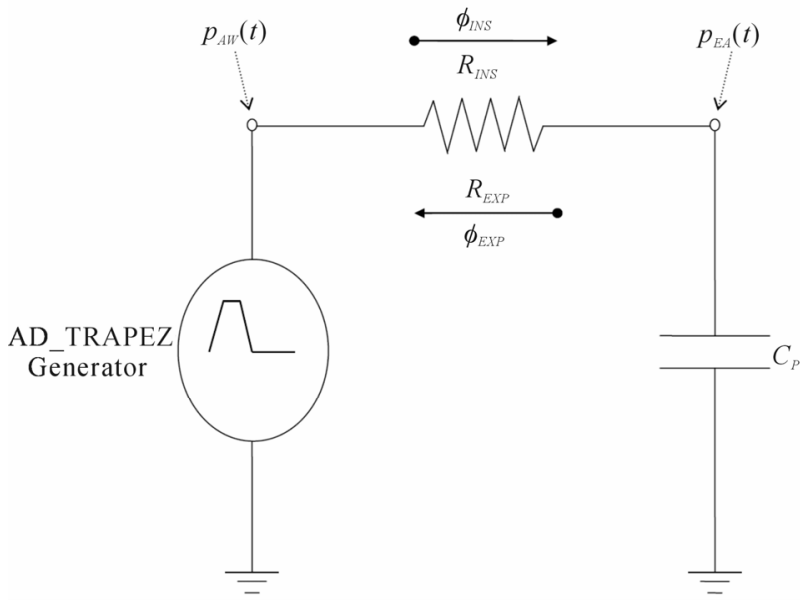

Figure 10. Electrical-equivalent circuit of AD_TRAPEZ generator connected to the patient's airways.

according to the results of $\S 3.3 .1$, if $t 1$ is set equal to $2 \tau_{I N S}\left(t 1=2 \tau_{I N S}\right)$, the following expressions result:

$$
\begin{aligned}
& p_{A W i}\left(t 1=2 \tau_{I N S}\right)=P I P=P E E P_{E X T}+2 k_{2 i} \tau_{I N S} \\
& v_{p i}\left(t 1=2 \tau_{I N S}\right)=F R C+k_{2 i} C_{P}\left[2 \tau_{I N S}-\tau_{I N S}\left(1-\mathrm{e}^{-2}\right)\right] \\
& \cong F R C+1.14 k_{2 i} C_{P} \tau_{I N S} \\
& \phi_{I N S}\left(t 1=2 \tau_{I N S}\right)=k_{2 i} C_{P}\left(1-\mathrm{e}^{-2}\right) \cong 0.86 k_{2 i} C_{P} \\
& p_{E A i}\left(t 1=2 \tau_{I N S}\right)=\frac{v_{p i}\left(t 1=2 \tau_{I N S}\right)}{C_{P}} \\
& \cong P E E P_{E X T}+1.14 k_{2 i} \tau_{I N S} \\
& \Delta p_{i}\left(t 1=2 \tau_{I N S}\right)=p_{A W i}\left(t 1=2 \tau_{I N S}\right)-p_{E A i}\left(t 1=2 \tau_{I N S}\right) \\
& \cong 0.86 k_{2 i} \tau_{I N S}
\end{aligned}
$$

According to AD_SQUARE analysis ( $§ 3.1)$, during the second interval of inspiration $\left(t 1 \leq t_{i} \leq t 1+t 2=T I\right)$, on account of initial conditions $\left(t_{i}=t 1\right)$ established by (116)-(120), the following expressions can be deduced:

$$
\begin{gathered}
p_{A W i}\left(t_{i}\right)=p_{A W i}(t 1)=P I P=P E E P_{E X T}+2 k_{2 i} \tau_{I N S} \\
p_{E A i}\left(t_{i}\right)=p_{A W i}(t 1)-\Delta p_{i}(t 1) \mathrm{e}^{-\frac{t_{i}-t 1}{\tau_{I N S}}} \\
\cong P E E P_{E X T}+k_{2 i} \tau_{I N S}\left(2-0.86 \mathrm{e}^{-\frac{t_{i}-t 1}{\tau_{I N S}}}\right) \\
v_{P i}\left(t_{i}\right)=p_{E A i}\left(t_{i}\right) C_{P} \\
\cong F R C+k_{2 i} C_{P} \tau_{I N S}\left(2-0.86 \mathrm{e}^{-\frac{t_{i}-t 1}{\tau_{I N S}}}\right)
\end{gathered}
$$

$$
\begin{aligned}
& \Delta p_{i}\left(t_{i}\right)=p_{A W i}\left(t_{i}\right)-p_{E A i}\left(t_{i}\right) \cong 0.86 k_{2 i} \tau_{I N S} \mathrm{e}^{-\frac{t_{i}-t 1}{\tau_{I N S}}} \\
& \phi_{I N S}\left(t_{i}\right)=\frac{\Delta p_{i}\left(t_{i}\right)}{R_{I N S}} \cong 0.86 k_{2 i} C_{P} \mathrm{e}^{-\frac{t_{i}-t 1}{\tau_{I N S}}}
\end{aligned}
$$

The functions $v_{P i}\left(t_{i}\right), \phi_{I N S}\left(t_{i}\right)$ and $p_{E A i}\left(t_{i}\right)$ are reported in Figure 11, Figure 12 and Figure 13, respectively.

If $t 2$ is set equal to $3 \tau_{I N S}\left(t 2=3 \tau_{I N S}\right)$ and from (113), the following conditions result:

$$
\begin{aligned}
& T I=5 \tau_{\text {INS }} \\
& t 2=1.5 t 1
\end{aligned}
$$

Condition (126) allows the best functional comparison between AD_SQUARE, AD_TRIANG and AD_TRAPEZ, while condition (127) represents the best trade-off for minimizing the final value of $\phi_{I N S}$ anyhow retaining a sufficient degree of smoothing on $\phi_{I N S}$ rising at the beginning of inspiration (Figure 12).

At the end of inspiration time $\left(t_{i}=T I\right)$ with an AD_TRAPEZ for which both (126) and (127) result, the following expressions can be deduced:

$$
\begin{aligned}
& p_{A W i}\left(T I=5 \tau_{I N S}\right)=p_{A W i}\left(t 1=2 \tau_{I N S}\right)=P I P \\
&=P E E P_{E X T}+2 k_{2 i} \tau_{I N S} \\
& p_{E A i}\left(T I=5 \tau_{I N S}\right)=P A P \\
&=P E E P_{E X T}+k_{2 i} \tau_{I N S}\left[2-0.86 \mathrm{e}^{-3}\right] \\
& \cong \\
& P E E P_{E X T}+2 k_{2 i} \tau_{I N S} \\
& v_{p i}\left(T I=5 \tau_{I N S}\right)=p_{E A i}\left(T I=5 \tau_{I N S}\right) C_{P} \\
& \cong F R C+2 k_{2 i} C_{P} \tau_{I N S} \\
& \Delta p_{i}\left(T I=5 \tau_{I N S}\right)=p_{A W i}\left(T I=5 \tau_{I N S}\right)-p_{E A i}\left(T I=5 \tau_{I N S}\right) \\
& \cong 0 \quad \\
& \phi_{I N S}\left(T I=5 \tau_{I N S}\right)=\frac{\Delta p_{i}\left(T I=5 \tau_{I N S}\right)}{R_{I N S}} \cong 0 \\
& V_{T I D}\left(T I=5 \tau_{I N S}\right)=v_{p i}\left(T I=5 \tau_{I N S}\right)-v_{p i}(0) \cong 2 k_{2 i} C_{P} \tau_{I N S}
\end{aligned}
$$

From both (12) and (133), $V_{M I N}$ for an inspiration time equal to $T I\left(V_{M I N}(T I)\right)$, results as follows:

$$
V_{\text {MIN }}(T I) \cong \frac{24 k_{2 i} \tau_{I N S}}{R_{I N S}+R_{E X P}}
$$

(134) takes into account that $T E$ is set equal to $5 \tau_{E X P}(\S$ 3.4.2).

According to our purpose, (131) and (132) establish the reduction to zero of both $\Delta p_{i}(T I)$ and $\phi_{I N S}(T I)$. 


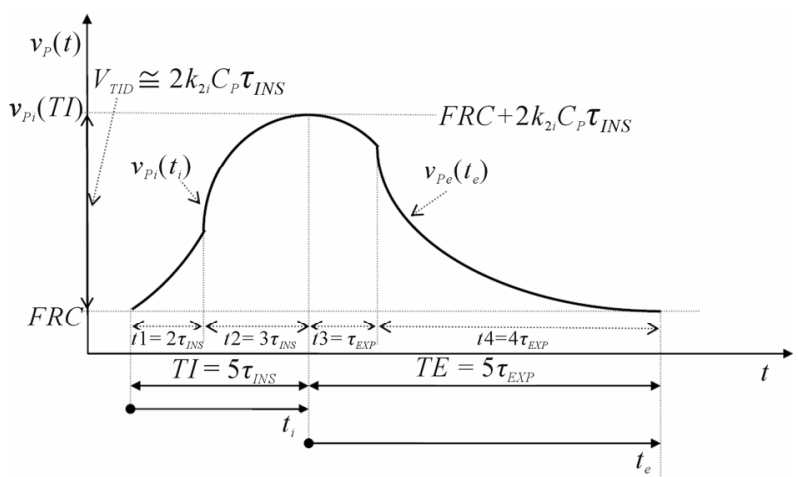

Figure 11. Lung volume $\left(v_{P}(t)\right)$ as a function of time $(t)$ resulting from the application of AD_TRAPEZ.

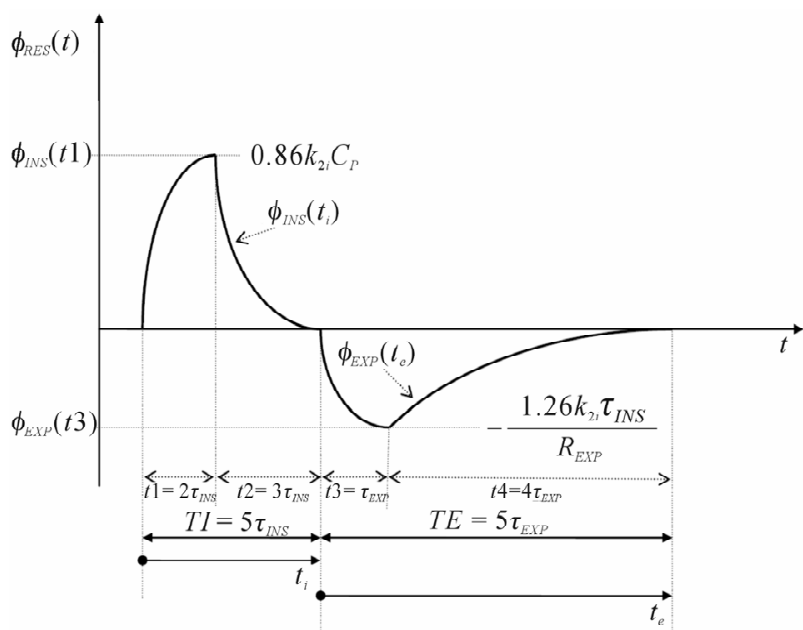

Figure 12. Respiratory airflow $\left(\phi_{R E S}(t)\right)$ as a function of time $(t)$ resulting from the application of AD_TRAPEZ.

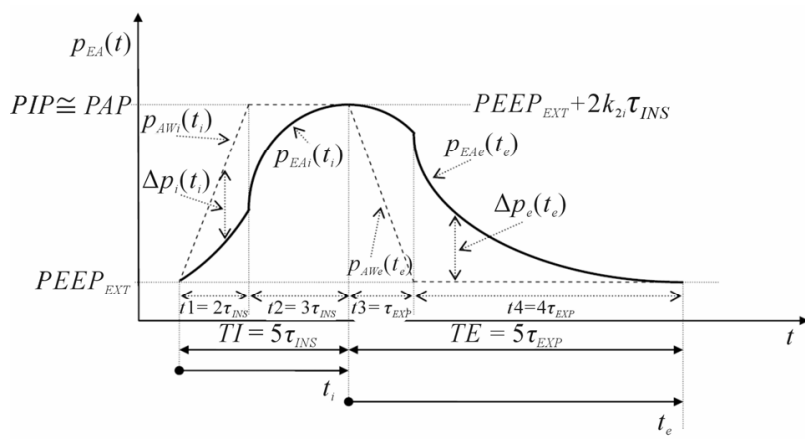

Figure 13. Endoalveolar pressure $\left(p_{E A}(t)\right)$ as a function of time $(t)$ resulting from the application of AD_TRAPEZ.

Expressions (116)-(134) provide the rationale for the optimization of ventilation control in AD_TRAPEZ during the time of inspiration.

$k_{2 i}$ value required for delivering in the same time (TI = $\left.5 \tau_{\text {INS }}\right)$ the same $V_{\text {TID }}$ as in AD_TRIANG $\left(k_{1 i}\right)$, can be determined by setting (133) equal to (42), as follows:

$$
k_{2 i} \cong 2 k_{1 i}
$$

Therefore, by comparing Eqs.37-43 with Eqs.116-134 in those the condition (135) has to be taken into account, the following consideration can be deduced. With the same TI, $V_{\text {TID }}$ and thus PAP values, $p_{A W}$ increasing slope of AD_TRAPEZ is practically doubled while the component of PIP above PEEP $P_{E X T}$ and the peak of $\phi_{I N S}\left(\phi_{I N S}\right.$ $\left(t_{i}=t 1\right)$ ), still independent on $R_{I N S}$, show a $20 \%$ reduction and a $72 \%$ increase, respectively, compared to those of AD_TRIANG. According to our purpose, $\Delta p_{i}(T I)$ and thus $\phi_{I N S}(T I)$ are both practically reduced to zero Figure 13 and Figure 12).

$k_{2 i}$ value required for delivering in the same time (TI= $\left.5 \tau_{\text {INS }}\right)$ the same $V_{\text {TID }}$ as in AD_SQUARE $\left(k_{\text {squ2i }}\right)$, can be determined by setting (133) equal to (11), obtaining the following expression:

$$
k_{\text {squ } 2 i} \cong \frac{P I-P E E P_{E X T}}{2 \tau_{I N S}} \cong 2 k_{\text {squ1i }}
$$

Under the last condition $\left(k_{2 i}=k_{s q u 2 i}\right)$, the following expressions result:

$$
\begin{aligned}
& P I P \cong P I \\
& P A P \cong P I \\
& P I P-P A P \cong 0 \\
& \phi_{I N S}(t 1) \cong \frac{0.43\left(P I-P E E P_{E X T}\right)}{R_{I N S}}
\end{aligned}
$$

So, the peak of $\phi_{I N S}$ and PIP required in AD_TRAPEZ for delivering in the same time $\left(T I=5 \tau_{I N S}\right)$ the same $V_{T I D}$ and thus for reaching the same PAP as in AD_SQUARE shows a $57 \%$ reduction and keeps equals to the upper $(P I)$ $p_{A W}$ constant level, respectively.

The diagnostic procedure has to be implemented as follows. The time required for reaching the end of transient inspiration time $\left(t_{T I}=5 \tau_{I N S}\right)$ cannot be precisely measured with AD_TRAPEZ, due to the influence of unknown $\tau_{I N S}$ on $t 1$ and $t 2$. The regular application of AD_TRIANG (§ 3.3.1) every few minutes represents the most suitable way to solve such problem. Once $t_{T I}$ has been evaluated in such a way, $\tau_{I N S}$ can be determined by means of (49). According to (118), for a given $k_{2 i}$ value, the monitoring of $\phi_{I N S}\left(t 1=2 \tau_{I N S}\right)$ leads to the determination of $C_{P}$, as follows:

$$
C_{P} \cong \frac{\phi_{I N S}\left(t 1=2 \tau_{I N S}\right)}{0.86 k_{2 i}}
$$

From (1), (49) and (141), $R_{I N S}$ can be determined as follows:

$$
R_{I N S}=\frac{\tau_{I N S}}{C_{P}} \cong \frac{0.86 t_{T I} k_{2 i}}{5 \phi_{I N S}\left(t 1=2 \tau_{I N S}\right)}
$$

Once $\tau_{I N S}, C_{P}$ and $R_{I N S}$ have been determined, TI should 
be set equal to the measured $t_{T I}\left(T I=t_{T I}\right)$ and $k_{2 i}$ regulation should be performed in order to fit the clinical requirements on PIP, PAP, $V_{T I D}$ or $V_{M I N}$ through (128), (129), (133) or (134), respectively. In particular, concerning with dual-control mode, $k_{2 i}$ values ensuring the pre-set $V_{\text {TID }}\left(k_{2 i T I D}\right)$ or $V_{\text {MIN }}\left(k_{2 i M I N}\right)$ can be determined from (133) or (134), as follows:

$$
\begin{aligned}
& k_{2 i T I D} \cong \frac{V_{T I D}}{2 C_{P} \tau_{I N S}} \\
& k_{2 i M I N} \cong \frac{V_{M I N}\left(R_{I N S}+R_{E X P}\right)}{24 \tau_{I N S}}
\end{aligned}
$$

$P I P$ values resulting from $V_{\text {TID }}\left(P I P_{T I D}\right)$ or $V_{M I N}\left(P I P_{M I N}\right)$ dual-control mode, can be obtained by inserting (143) or (144) into (128), as follows:

$$
\begin{aligned}
P I P_{T I D} & \cong P E E P_{E X T}+\frac{V_{T I D}}{C_{P}} \\
P I P_{M I N} & \cong P E E P_{E X T}+\frac{V_{M I N}\left(R_{I N S}+R_{E X P}\right)}{12}
\end{aligned}
$$

In the same way as (54) and (55), (145) and (146) show that $P I P_{T I D}$ or $P I P_{M I N}$ are independent on $R_{R E S}$ or $C_{P}$, respectively. That is, again, extremely relevant from both physiopathological and clinical point of view since an increase of $R_{R E S}$ (obstructive process) or a reduction of $C_{P}$ (restrictive process) does not affect the maximum $p_{A W}$ value reached for dual-control mode with pre-set $V_{\text {TID }}$ or $V_{M I N}$, respectively. Moreover, by comparing (145) and (146) with (54) and (55), respectively, the component of PIP $_{\text {TID }}$ and PIP $P_{M I N}$ above PEEP $P_{E X T}$ of AD TRAPEZ both show a $20 \%$ reduction with respect to those of $A D$ TRIANG.

The compensation procedure is required in order to assimilate ALVS with an ideal $p_{A W}$ generator providing for a real AD_TRAPEZ, i.e. trapezoidal waveform as $p_{A W}$ excitation insensitive to patient's respiratory characteristics and ventilator settings through a proper trapezoidal waveform of external resistance $\left(R_{E X T}\right)$ of ALVS which controls $p_{A W}$ [9]. The electrical-equivalent network of ALVS is shown in Figure 7. With the same approach described in $\S 3.3 .1$, if $\phi_{E X T}$ is stabilized on constant steady $\phi_{\text {EXTO }}$ value, AD_TRAPEZ can be obtained by modeling $R_{E X T}$ waveform during inspiration $\left(R_{E X T i}\left(t_{i}\right)\right)$ as linear increasing of $R_{E X T}$ from $R_{E X T 0}$ to $R_{E X T}^{*}$ in the first interval $\left(0 \leq t_{i} \leq t 1\right)$ and as constant keeping of $R_{E X T}$ on $R_{E X T}^{*}$ in the second interval $\left(t 1 \leq t_{i} \leq t 1+t 2\right)$, respectively, while during expiration $\left(R_{\text {EXTe }}\left(t_{e}\right)\right)$ as linear decreasing of $R_{E X T}$ from $R_{E X T}^{*}$ to $R_{E X T 0}$ in the first interval $\left(0 \leq t_{e} \leq t 3\right)$ and as constant keeping of $R_{E X T}$ on $R_{E X T 0}$ in the second interval $\left(t 3 \leq t_{e} \leq t 3+t 4\right)$, respectively. In particular, concerning with dual-control mode, $R_{E X T}^{*}$ values ensuring the pre-set $V_{T I D}\left(R_{E X T}^{* T I D}\right)$ or $V_{M I N}\left(R_{E X T}^{* M I N}\right)$ can be obtained by inserting (145) or (146) into (61), respectively, and considering (9), as follows:

$$
\begin{aligned}
& R_{E X T}^{* T I D} \cong R_{E X T 0}\left(1+\frac{V_{T I D}}{F R C}\right) \\
& R_{E X T}^{* M I N} \cong R_{E X T 0}\left[1+\frac{V_{M I N}\left(R_{I N S}+R_{E X P}\right)}{12 P E E P_{E X T}}\right]
\end{aligned}
$$

So, $R_{E X T}\left(t_{i}\right)$ to be implemented for dual-control mode during the first $\left(0 \leq t_{i} \leq t 1\right)$ and second $\left(t 1 \leq t_{i} \leq T I\right)$ interval of inspiration $\left(0 \leq t_{i} \leq T I\right)$ with pre-set $V_{T I D}$ $\left(R_{E X T}\left(t_{i}\right)^{T I D}\right)$ or for $V_{M I N}\left(R_{E X T}\left(t_{\mathrm{i}}\right)^{M I N}\right)$, assume the following expressions:

$$
\begin{gathered}
R_{E X T}\left(0 \leq t_{i} \leq t 1\right)^{T I D} \cong R_{E X T 0}\left(1+\frac{V_{T I D} t_{i}}{2 F R C \tau_{I N S}}\right) \\
R_{E X T}\left(0 \leq t_{i} \leq t 1\right)^{M I N} \cong R_{E X T 0}\left[1+\frac{V_{M I N}\left(R_{I N S}+R_{E X P}\right) t_{i}}{24 R_{I N S} F R C}\right] \\
R_{E X T}\left(t 1 \leq t_{i} \leq T I\right)^{T I D} \equiv R_{E X T}^{* T I D} \\
R_{E X T}\left(t 1 \leq t_{i} \leq T I\right)^{M I N} \equiv R_{E X T}^{* M I N}
\end{gathered}
$$

According to (66), the stabilization of $\phi_{E X T}$ during inspiration $\left(\phi_{E X T i}\left(t_{i}\right)\right)$ on $\phi_{E X T 0}$ can be carried out by proper modeling $\phi_{V E N}$ waveform during inspiration $\left(\phi_{V E N i}\left(t_{i}\right)\right)$.

Concerning the first interval of inspiration $\left(0 \leq t_{i} \leq t 1\right)$, on account of (20) and (31) with $k_{2 i}$ instead of $k_{1 i}$ as well as (57), (66) and (68), the following expressions result:

$$
\begin{gathered}
\phi_{V E N i}\left(t_{i}\right)=\frac{P_{G}}{R_{G 0}}+k_{2 i} C_{P}\left(1-\mathrm{e}^{-\frac{t_{i}}{\tau_{I N S}}}\right) \\
R_{G i}\left(t_{i}\right)=\frac{P_{G}-P E E P_{E X T}-k_{2 i} t_{i}}{\frac{P_{G}}{R_{G 0}}+k_{2 i} C_{P}\left(1-\mathrm{e}^{-\frac{t_{i}}{\tau_{I N S}}}\right)}
\end{gathered}
$$

With $k_{2 i}$ instead of $k_{1 i}$, (70)-(77) can be retained and in particular, (74) assumes the following expression:

$$
\begin{aligned}
R_{G i}\left(t_{i}\right) \cong & \frac{P_{G}}{\frac{P_{G}}{R_{G 0}}+k_{2 i} C_{P}\left(1-\mathrm{e}^{-\frac{t_{i}}{\tau_{I N S}}}\right)} \\
= & \frac{R_{G 0} P E E P_{E X T}}{P E E P_{E X T}+k_{2 i} R_{E X T 0} C_{P}\left(1-\mathrm{e}^{-\frac{t_{i}}{\tau_{I N S}}}\right)}
\end{aligned}
$$

Concerning the second interval of inspiration $\left(t 1 \leq t_{i} \leq\right.$ $t 1+t 2=T I)$, on account of (121) and (125) as well as (57), (66) and (68), the following expressions result: 


$$
\begin{aligned}
\phi_{V E N i}\left(t_{i}\right)= & \frac{P_{G}}{R_{G 0}}+0.86 k_{2 i} C_{P} \mathrm{e}^{-\frac{t_{i}-t 1}{\tau_{I N S}}} \\
R_{G i}\left(t_{i}\right)= & \frac{P_{G}-P E E P_{E X T}-2 k_{2 i} \tau_{I N S}}{\frac{P_{G}}{R_{G 0}}+0.86 k_{2 i} C_{P} \mathrm{e}^{-\frac{t_{i}-t 1}{\tau_{I N S}}}}
\end{aligned}
$$

On account of (73) and (58), (157) reduces to the following expressions:

$$
\begin{aligned}
R_{G i}\left(t_{i}\right) \cong & \frac{P_{G}}{\frac{P_{G}}{R_{G 0}}+0.86 k_{2 i} C_{P} \mathrm{e}^{-\frac{t_{i}-t 1}{\tau_{I N S}}}} \\
= & \frac{R_{G 0} P E E P_{E X T}}{P E E P_{E X T}+0.86 k_{2 i} R_{E X T 0} C_{P} \mathrm{e}^{-\frac{t_{i}-t 1}{\tau_{I N S}}}}
\end{aligned}
$$

The function $R_{G i}\left(t_{i}\right)$ is reported in Figure 14.

So that, the implementation of (155) and (158) during the first $\left(0 \leq t_{i} \leq t 1\right)$ and second $\left(t 1 \leq t_{i} \leq t 1+t 2=T I\right)$ intervals, respectively, ensures the compensation procedure to be carried out during the whole inspiration time $(0$ $\left.\leq t_{i} \leq T I\right)$.

\subsubsection{Expiration Time}

Concerning to the first interval of expiration $\left(0 \leq t_{e} \leq t 3\right)$, according to (128) and to Figure 9, AD_TRAPEZ requires the following expression of $p_{\text {AWe }}\left(t_{e}\right)$ :

$$
p_{\text {AWe }}\left(t_{e}\right)=P E E P_{E X T}+2 k_{2 i} \tau_{\text {INS }}\left(1-\frac{t_{e}}{t 3}\right)
$$

Considering both (3) and (9) as well as by inserting both (159) and (80) into (78), the following equation results:

$$
\begin{aligned}
& \operatorname{PEEP}_{E X T}+2 k_{2 i} \tau_{I N S}\left(1-\frac{t_{e}}{t 3}\right) \\
& -R_{E X P} \frac{\mathrm{d} v_{P e}\left(t_{e}\right)}{\mathrm{d} t_{e}}-\frac{v_{P e}\left(t_{e}\right)}{C_{P}}=0
\end{aligned}
$$

In order to solve Eq.160, i.e. to find out the transient and steady expressions of $v_{P e}\left(t_{e}\right)$, it is useful to transform it from time $\left(t_{e}\right)$ to Laplace $(s)$ variable domains, as follows:

$$
\begin{aligned}
& \frac{P E E P_{E X T}+2 k_{2 i} \tau_{I N S}}{s}-\frac{\frac{2 k_{2 i} \tau_{I N S}}{t 3}}{s^{2}} \\
& -R_{E X P}\left[s v_{P e}(s)-v_{P e}(0)\right]-\frac{v_{P e}(s)}{C_{P}}=0
\end{aligned}
$$

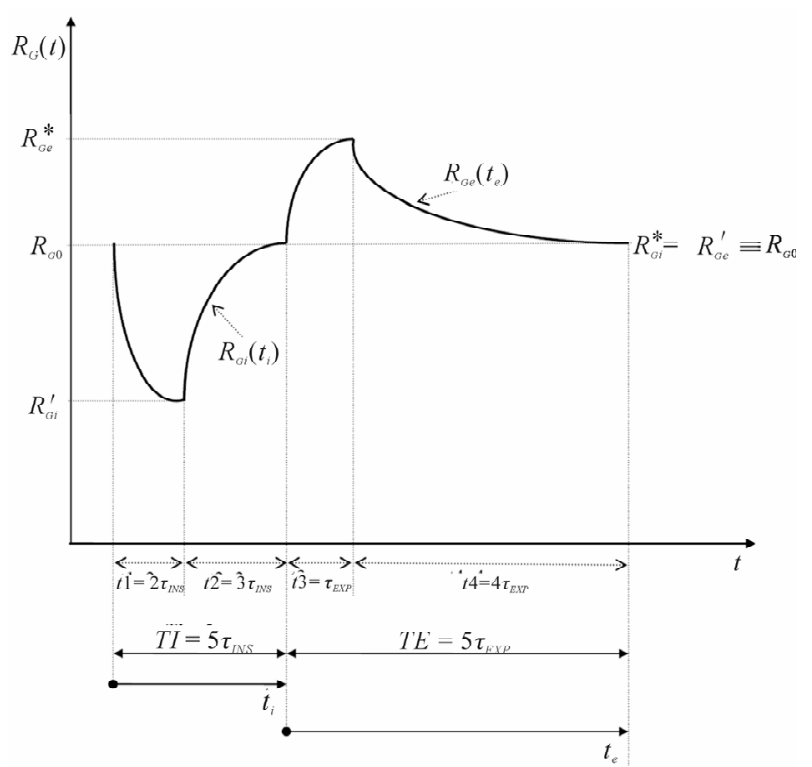

Figure 14. Internal resistance $\left(R_{G}(t)\right)$ of ALVS generator as a function of time $(t)$ to be implemented for performing the compensation procedure in AD_TRAPEZ.

From (2) and (130), on account of continuity condition on $v_{P}$ when the switching between inspiration and expiration takes place $\left(v_{P e}(0) \equiv v_{P i}(T I)\right)$, the solution of $(161)$ consists in the following expression (see * in the end):

Eq.162 can be properly decomposed as follows:

$$
v_{P e}(s)=\frac{A}{s^{2}}+\frac{B}{s}+\frac{C}{s+\frac{1}{\tau_{E X P}}}
$$

The unknown constants $\mathrm{A}, \mathrm{B}$ and $\mathrm{C}$ can be determined by setting Eq.162 equal to Eq.163, resulting as follows:

$$
\begin{aligned}
A & =-\frac{2 k_{2 i} C_{P} \tau_{I N S}}{t 3} \\
B & =C_{P}\left[P E E P_{E X T}+2 k_{2 i} \tau_{I N S}\left(1+\frac{\tau_{E X P}}{t 3}\right)\right] \\
& =F R C+2 k_{2 i} C_{P} \tau_{I N S}\left(1+\frac{\tau_{E X P}}{t 3}\right) \\
C & =-\frac{2 k_{2 i} C_{P} \tau_{I N S} \tau_{E X P}}{t 3}
\end{aligned}
$$

Finally, by inserting (164), (165) and (166) into (163),

$$
* v_{P e}(s)=\frac{\left(F R C+2 k_{2 i} C_{P} \tau_{I N S}\right) s^{2}+\left(\frac{P E E P_{E X T}+2 k_{2 i} \tau_{I N S}}{R_{E X P}}\right) s-\frac{2 k_{2 i} \tau_{I N S}}{t 3 R_{E X P}}}{s^{2}\left(s+\frac{1}{\tau_{E X P}}\right)}
$$


the following expression results:

$$
\begin{aligned}
& v_{P e}(s)=-\frac{\frac{2 k_{2 i} C_{P} \tau_{I N S}}{t 3}}{s^{2}} \\
& +\frac{C_{P}\left[P E E P_{E X T}+2 k_{2 i} \tau_{I N S}\left(1+\frac{\tau_{E X P}}{t 3}\right)\right]}{s} \\
& -\frac{\frac{2 k_{2 i} C_{P} \tau_{I N S} \tau_{E X P}}{t 3}}{s+\frac{1}{\tau_{E X P}}}
\end{aligned}
$$

According to the Inverse Laplace Transform of (167), the function $v_{P e}\left(t_{e}\right)$ assumes the following expression:

$$
\begin{aligned}
v_{P e}\left(t_{e}\right)= & -\frac{2 k_{2 i} C_{P} \tau_{I N S}}{t 3} t_{e}+F R C \\
& +2 k_{2 i} C_{P} \tau_{I N S}\left(1+\frac{\tau_{E X P}}{t 3}\right) \\
& -\frac{2 k_{2 i} C_{P} \tau_{I N S} \tau_{E X P}}{t 3} \mathrm{e}^{-\frac{t_{e}}{\tau_{E X P}}}
\end{aligned}
$$

The functions $\phi_{E X P}\left(t_{e}\right)$ and $p_{E A e}\left(t_{e}\right)$ can be determined considering (80) and (3), respectively, as follows:

$$
\begin{aligned}
\phi_{E X P}\left(t_{e}\right)= & \frac{2 k_{2 i} C_{P} \tau_{I N S}}{t 3}\left(1-\mathrm{e}^{-\frac{t_{e}}{\tau_{E X P}}}\right) \\
p_{E A e}\left(t_{e}\right)= & -\frac{2 k_{2 i} \tau_{I N S}}{t 3} t_{e}+P E E P_{E X T} \\
& +2 k_{2 i} \tau_{I N S}\left(1+\frac{\tau_{E X P}}{t 3}\right) \\
& -\frac{2 k_{2 i} \tau_{I N S} \tau_{E X P}}{t 3} \mathrm{e}^{-\frac{t_{e}}{\tau_{E X P}}}
\end{aligned}
$$

The difference between $p_{\text {AWe }}\left(t_{e}\right)$ and $p_{\text {EAe }}\left(t_{e}\right)\left(\Delta p_{e}\left(t_{e}\right)\right)$ can be determined from both (159) and (170), as follows (Figure 13):

$$
\Delta p_{e}\left(t_{e}\right)=-\frac{2 k_{2 i} \tau_{I N S} \tau_{E X P}}{t 3}\left(1-\mathrm{e}^{-\frac{t_{e}}{\tau_{E X P}}}\right)
$$

The same result of (171) could also be obtained by inserting (169) into (78).

Considering (168), (169) and (170) at the beginning of expiration time $\left(t_{e}=0\right)$, the following expressions result:

$$
\begin{gathered}
v_{p e}(0)=F R C+2 k_{2 i} C_{P} \tau_{I N S}=v_{p i}(T I) \\
\phi_{E X P}(0)=0 \\
p_{E A e}(0)=P E E P_{E X T}+2 k_{2 i} \tau_{I N S}=p_{E A i}(T I)
\end{gathered}
$$

(172) and (174) fit well (130) and (129), respectively, according to continuity condition required at the transition between the end of inspiration and the beginning of expiration (Figure 11 and Figure 13). Moreover, according to our purpose, (173) together with (132) establish the real elimination of $\phi_{R E S}$ discontinuity occurring in coincidence with such transition and the reduction to zero of $\phi_{E X P}(0)$ (Figure 12).

If $t 3$ is set equal to $\tau_{E X P}\left(t 3=\tau_{E X P}\right)$, the following expressions result:

$$
\begin{aligned}
& p_{A W e}\left(t 3=\tau_{E X P}\right)=P E E P_{E X T} \\
& v_{P e}\left(t 3=\tau_{E X P}\right) \cong F R C+1.26 k_{2 i} C_{P} \tau_{I N S} \\
& \phi_{E X P}\left(t 3=\tau_{E X P}\right) \cong \frac{1.26 k_{2 i} \tau_{I N S}}{R_{E X P}} \\
& p_{E A e}\left(t 3=\tau_{E X P}\right) \cong P E E P_{E X T}+1.26 k_{2 i} \tau_{I N S} \\
& \Delta p_{e}\left(t 3=\tau_{E X P}\right) \cong-1.26 k_{2 i} \tau_{I N S}
\end{aligned}
$$

According to AD_SQUARE analysis ( $\$ 3.1$ ), during the second interval of expiration $\left(t 3 \leq t_{e} \leq t 3+t 4=T E\right)$, on account of initial conditions $\left(t_{e}=t 3\right)$ established by (175)-(179), the following expressions can be deduced:

$$
\begin{gathered}
p_{\text {AWe }}\left(t_{e}\right)=p_{\text {AWe }}(t 3)=P E E P_{E X T} \\
p_{E A e}\left(t_{e}\right)=p_{A W e}(t 3)-\left[\Delta p_{e}(t 3) \mathrm{e}^{-\frac{t_{e}-t 3}{\tau_{E X P}}}\right] \\
\cong P E E P_{E X T}+1.26 k_{2 i} \tau_{I N S} \mathrm{e}^{-\frac{t_{e}-t 3}{\tau_{E X P}}} \\
v_{P e}\left(t_{e}\right)=p_{E A e}\left(t_{e}\right) C_{P} \cong F R C+1.26 k_{2 i} C_{P} \tau_{I N S} \mathrm{e}^{-\frac{t_{e}-t 3}{\tau_{E X P}}} \\
\Delta p_{e}\left(t_{e}\right)=\Delta p_{e}(t 3) \mathrm{e}^{-\frac{t_{e}-t 3}{\tau_{E X P}}} \cong-1.26 k_{2 i} \tau_{I N S} \mathrm{e}^{-\frac{t_{e}-t 3}{\tau_{E X P}}} \\
\phi_{E X P}\left(t_{e}\right)=-\frac{\Delta p_{e}\left(t_{e}\right)}{R_{E X P}}=\frac{1.26 k_{2 i} \tau_{I N S}}{R_{E X P}} \mathrm{e}^{-\frac{t_{e}-t 3}{\tau_{E X P}}}
\end{gathered}
$$

The functions $v_{P e}\left(t_{e}\right), \phi_{E X P}\left(t_{e}\right)$ and $p_{E A e}\left(t_{e}\right)$ are reported in Figure 11, Figure 12 and Figure 13, respectively.

If $t 4$ is set equal to $4 \tau_{E X P}\left(t 4=4 \tau_{E X P}\right)$ and from (115), the following conditions result:

$$
\begin{aligned}
& T E=5 \tau_{I N S} \\
& t 4=4 t 3
\end{aligned}
$$

Condition (185) allows the best functional comparison between AD_SQUARE, AD_TRIANG and AD_TRAPEZ, while condition (186) represents the best trade-off for minimizing the final value of $\phi_{E X P}$ along with the increase of MAP (see (203)) and MEP (see (204)), anyhow retaining a sufficient degree of smoothing on $\phi_{E X P}$ 
raising at the beginning of expiration (Figure 12).

At the end of expiration time $\left(t_{e}=T E\right)$ with an AD_TRAPEZ for which both conditions (185) and (186) result, the following expressions can be deduced:

$$
\begin{aligned}
& p_{A W e}\left(T E=5 \tau_{E X P}\right)=p_{A W e}\left(t_{3}\right)=P E E P_{E X T} \\
& p_{E A e}\left(T E=5 \tau_{E X P}\right) \cong P E E P_{E X T}+1.26 k_{2 i} \tau_{I N S} \mathrm{e}^{-4} \cong P E E P_{E X T} \\
& v_{P e}\left(T E=5 \tau_{E X P}\right)=p_{E A e}(T E) C_{P} \\
& \cong F R C+1.26 k_{2 i} C_{P} \tau_{I N S} \mathrm{e}^{-4} \\
& \cong F R C \\
& \Delta p_{e}\left(T E=5 \tau_{E X P}\right) \cong-1.26 k_{2 i} \tau_{I N S} \mathrm{e}^{-4} \cong 0 \\
& \phi_{E X P}\left(T E=5 \tau_{E X P}\right) \cong \frac{1.26 k_{2 i} \tau_{I N S} \mathrm{e}^{-4}}{R_{E X P}} \cong 0
\end{aligned}
$$

(189) and (188) fit well (34) and (36), respectively, according to continuity condition required at the transition between the end of every expiration and the beginning of the following inspiration (Figure 11 and Figure 13). Moreover, according to our purpose, (191) together with (35) and (190) establish the real elimination of $\phi_{R E S}$ discontinuity occurring in coincidence with such transition and the reduction to zero of both $\Delta p_{e}(T E)$ and $\phi_{E X P}(T E)$ (Figure 12 and Figure 13). Finally, from (177) together with (93), the peak of $\phi_{E X P}\left(\phi_{E X P}\left(t_{\mathrm{e}}=t 3\right)\right)$ shows a $37 \%$ reduction compared to those of $\mathrm{AD}$ TRIANG and $\mathrm{AD}$ $\operatorname{SQUARE}\left(\phi_{\text {EXP }}\left(t_{e}=0\right)\right)$.

Therefore, the waveforms reported in Figure 11, Figure 12 and Figure 13 compared to those reported in Figure 4, Figure 5 and Figure 6, respectively, show clearly that AD_TRAPEZ induces a more physiological reaction than $A D_{-}$TRIANG. This is essensially due to the elimination in AD_TRAPEZ of discontinuity on $\phi_{R E S}$ occurring in AD_TRIANG when the switching between inspiration and expiration takes place.

The diagnostic procedure has to be implemented as follows. The time required for reaching the end of transient expiration time $\left(t_{T E}=5 \tau_{E X P}\right)$ cannot be precisely measured with AD_TRAPEZ, due to the influence of unknown $\tau_{E X P}$ on $t \overline{3}$ and $t 4$. The regular application of AD_TRIANG $(\$ 3.3 .2)$ every few minutes represents the most suitable way to solve such problem. Once $t_{T E}$ has been evaluated in such a way, $\tau_{E X P}$ can be determined by means of (99). According to (177), for a given $k_{2 i}$ and $\tau_{I N S}$ values, the monitoring of $\phi_{E X P}\left(t 3=\tau_{E X P}\right)$ leads to the determination of $R_{E X P}$, as follows:

$$
R_{E X P} \cong \frac{1.26 k_{2 i} \tau_{I N S}}{\phi_{E X P}\left(t 3=\tau_{E X P}\right)}
$$

From (2), (99) and (192), $C_{P}$ can be determined as follows:

$$
C_{P}=\frac{\tau_{E X P}}{R_{E X P}} \cong \frac{t_{T E} \phi_{E X P}\left(t 3=\tau_{E X P}\right)}{6.3 k_{2 i} \tau_{I N S}}
$$

(193) can be employed for confirming the result obtained with (141). Once $\tau_{E X P}, R_{E X P}$ and $C_{P}$ have been determined, $T E$ should be set equal to the measured $t_{T E}\left(T E=t_{T E}\right)$.

So, considering (58), (133), (134), (159) and (180), $R_{E X T}\left(t_{e}\right)$ to be implemented for dual-control mode during the first $\left(0 \leq t_{e} \leq t 3\right)$ and second $\left(t 3 \leq t_{e} \leq T E\right)$ interval of expiration $\left(0 \leq t_{e} \leq T E\right)$ with pre-set $V_{T I D}\left(R_{E X T}\left(\mathrm{t}_{\mathrm{e}}\right)^{T I D}\right)$ or $V_{M I N}\left(R_{E X T}\left(t_{e}\right)^{M I N}\right)$, assumes the following expressions:

$$
\begin{aligned}
& R_{E X T}\left(0 \leq t_{e} \leq t 3\right)^{T I D} \cong R_{E X T 0}\left[1+\frac{V_{T I D}}{F R C}\left(1-\frac{t_{e}}{t 3}\right)\right] \quad(194) \\
& R_{E X T}\left(0 \leq t_{e} \leq t 3\right)^{M I N} \cong R_{E X T 0}\left\{1+\frac{V_{M I N}\left(R_{I N S}+R_{E X P}\right)}{12 P E E P_{E X T}}\left[1-\frac{t_{e}}{t 3}\right]\right\} \\
& R_{E X T}\left(t 3 \leq t_{e} \leq T E\right)^{T I D}=R_{E X T}\left(t 3 \leq t_{e} \leq T E\right)^{M I N} \equiv R_{E X T 0}
\end{aligned}
$$

According to (103), the compensation procedure, i.e. the stabilization of $\phi_{E X T}$ during expiration $\left(\phi_{E X T e}\left(t_{e}\right)\right)$ on $\phi_{\text {EXT0 }}$ can be carried out by proper modeling $\phi_{V E N}$ waveform during expiration $\left(\phi_{V E N e}\left(t_{e}\right)\right)$.

Concerning the first interval of expiration $\left(0 \leq t_{e} \leq t 3\right)$, on account of (57), (159) and (169) as well as (103) and (105), the following expressions result:

$$
\begin{aligned}
\phi_{V E N e}\left(t_{e}\right) & =\frac{P_{G}}{R_{G 0}}-\frac{2 k_{2 i} C_{P} \tau_{I N S}}{t 3}\left(1-\mathrm{e}^{-\frac{t_{e}}{\tau_{E X P}}}\right) \\
R_{G e}\left(t_{e}\right) & =\frac{P_{G}-P E E P_{E X T}-2 k_{2 i} \tau_{I N S}\left(1-\frac{t_{e}}{t 3}\right)}{\frac{P_{G}}{R_{G 0}}-\frac{2 k_{2 i} C_{P} \tau_{I N S}}{t 3}\left(1-\mathrm{e}^{-\frac{t_{e}}{\tau_{E X P}}}\right)}
\end{aligned}
$$

On account of both (73) and (58), (198) reduces to the following expression:

$$
\begin{aligned}
R_{G e}\left(t_{e}\right) \cong & \frac{P_{G}}{\frac{P_{G}}{R_{G 0}}-\frac{2 k_{2 i} C_{P} \tau_{I N S}}{t 3}\left(1-\mathrm{e}^{-\frac{t_{e}}{\tau_{E X P}}}\right)} \\
= & \frac{R_{G 0} P E E P_{E X T}}{P E E P_{E X T}-\frac{2 k_{2 i} R_{E X T 0} C_{P} \tau_{I N S}}{t 3}\left(1-\mathrm{e}^{-\frac{t_{e}}{\tau_{E X P}}}\right)}
\end{aligned}
$$


Concerning the second interval of expiration $\left(t 3 \leq t_{e} \leq\right.$ $t 3+t 4=T E)$, on account of (57), (180) and (184) as well as (103) and (105), the following expressions result:

$$
\begin{gathered}
\phi_{V E N e}\left(t_{e}\right)=\frac{P_{G}}{R_{G 0}}-\frac{1.26 k_{2 i} \tau_{I N S}}{R_{E X P}}\left(1-\mathrm{e}^{-\frac{t_{e}-t 3}{\tau_{E X P}}}\right) \\
R_{G e}\left(t_{e}\right)=\frac{P_{G}-P E E P_{E X T}}{\frac{P_{G}}{R_{G 0}}-\frac{1.26 k_{2 i} \tau_{I N S}}{R_{E X P}}\left(1-\mathrm{e}^{-\frac{t_{e}-t 3}{\tau_{E X P}}}\right)}
\end{gathered}
$$

On account of both (60) and (58), (201) reduces to the following expressions:

$$
\begin{aligned}
R_{G e}\left(t_{e}\right) \cong \frac{P_{G}}{\frac{P_{G}}{R_{G 0}}-\frac{1.26 k_{2 i} \tau_{I N S}}{R_{E X P}}\left(1-\mathrm{e}^{-\frac{t_{e}-t 3}{\tau_{E X P}}}\right)} \\
=\frac{R_{G 0} P E E P_{E X T}}{P E E P_{E X T}-\frac{1.26 k_{2 i} R_{E X T 0} \tau_{I N S}}{R_{E X P}}\left(1-\mathrm{e}^{-\frac{t_{e}-t 3}{\tau_{E X P}}}\right)}
\end{aligned}
$$

The function $R_{G e}\left(t_{e}\right)$ is reported in Figure 14 .

So that, the implementation of (199) and (202) during the first $\left(0 \leq t_{e} \leq t 3\right)$ and second $\left(t 3 \leq t_{e} \leq t 3+t 4=T E\right)$ intervals, respectively, ensures the compensation procedure to be carried out during the whole expiration time ( 0 $\left.\leq t_{e} \leq T E\right)$.

From Figure 9, Figure 13, (12), (15), (16), (116), (119), (121), (122), (136), (159), (170), (180) and (181), it is easy to demonstrate that $M A P$ and $M E P$ values of $\mathrm{AD}_{-}$ TRAPEZ $\left(M A P_{\text {tra }}\right.$ and $\left.M E P_{\text {tra }}\right)$ result as follows:

$$
\begin{aligned}
M P_{t r a} & =P E E P_{E X T} \\
& +\frac{\left(P I-P E E P_{E X T}\right)\left(0.8 \tau_{I N S}+0.1 \tau_{E X P}\right)}{\left(\tau_{I N S}+\tau_{E X P}\right)} \\
M E P_{t r a} \cong & P E E P_{E X T} \\
+ & \frac{\left(P I-P E E P_{E X T}\right)\left(0.604 \tau_{I N S}+0.297 \tau_{E X P}\right)}{\left(\tau_{I N S}+\tau_{E X P}\right)}
\end{aligned}
$$

If the ratio between $\tau_{E X P}$ and $\tau_{I N S}$ is estimated twice, in comparison with (17) and (18), (203) and (204) show that $M A P_{\text {tra }}$ and $M E P_{\text {tra }}$ assume quite the same values of $M A P_{\text {squ }}$ and $M E P_{\text {squ }}$, respectively.

\section{DISCUSSION AND CONCLUSIONS}

The promising experimental results, according to theo- retical ones, carried out in a previous work with the Advanced Lung Ventilation System (ALVS), set for applying a real square waveform as airways pressure excitation to a proper lung simulator reproducing the steady and linear respiratory mechanics of anaesthetized or severe brain injured patients, have suggested and motivated the present work.

It consists in the theoretical study in the field of assisted/controlled ventilation with advanced and improved-shape waveforms as airways pressure excitation for the optimization of controlled breathings applied to patients the respiratory mechanics of which can be assumed steady and linear. Advanced means insensitive to patient (load) breathing activity as well as to ventilator settings. Improved-shape intends in comparison to conventional square waveform for a progressive approaching to physiological transpulmonary pressure waveform producing a more suitable reaction of patient, i.e. a more realistic approximation of respiratory airflow and endoalveolar pressure waveforms to physiological ones.

The problem to be solved has been the proper smoothing of respiratory airflow vertical discontinuities occurring at the beginning of both inspiration and expiration when the respiratory airflow is reversed, as response to upward and downward airways pressure vertical transitions characteristic of square waveform. So that, the elimination of such airways pressure vertical transitions is the most relevant change to be applied on square waveform as airways pressure excitation. For the purpose, two waveforms of different geometrical shape (triangular and trapezoidal) as airways pressure excitation which progressively approach the best solution have been considered.

The results show that the application of both the diagnostic and compensation procedures together with the setting of the time of inspiration and expiration equal to five times the inspiratory and expiratory time constants, respectively, ensure the optimization of the ventilation control in all cases with the following different functional implications.

Advanced triangular (AD_TRIANG) and trapezoidal (AD_TRAPEZ) waveforms have been considered in comparison to conventional advanced square waveform (AD_SQUARE) as airways pressure excitation. The geometrical parameters of AD_TRAPEZ has been optimized in such a way the resulting respiratory airflow waveform does not show any vertical discontinuity approximating as much as possible the smoothed shape of physiological waveform as well as keeping quite the same values of mean airways (MAP) and endoalveolar pressures $(M E P)$ of AD_SQUARE.

AD_SQUARE shows a low physiological profile due to the presence of two different considerable disconti- 
nuities on respiratory airflow waveform occurring when the switching between inspiration and expiration and vice versa, take place. Concerning with dual-control mode, tidal $\left(V_{T I D}\right)$ and minute $\left(V_{M I N}\right)$ volumes are independent on respiratory resistance and lung compliance, respectively. That is extremely relevant from physiological, clinical and engineering point of view because an increase of respiratory resistance (obstructive process) or a reduction of lung compliance (restrictive process) does not affect the control of $V_{T I D}$ or $V_{M I N}$, respectively.

AD_TRIANG eliminates the discontinuity on respiratory airflow waveform between expiration and following inspiration but increases the amount of the discontinuity on respiratory airflow between inspiration and expiration. Moreover, the diagnostic procedure is available for accurate results. Keeping the same values of inspiration (TI) and expiration $(T E)$ times and thus of breathing frequency $(F R)$, as well as of external positive end expiratory pressure $\left(P E E P_{E X T}\right)$ and $V_{T I D}$ and thus of peak endoalveolar pressure $(P A P)$ with regard to AD_SQUARE, the peak inspiratory airways pressure $(P I P)$ increases less than $25 \%$ while the components of MAP and MEP above $P E E P_{E X T}$ show a $37.5 \%$ and $31.3 \%$ reduction, respectively.

AD TRAPEZ eliminates both the discontinuities on respiratory airflow waveform providing for the desired physiological shape of both respiratory airflow and endoalveolar pressure waveforms. Unfortunately, the diagnostic procedure is not available for accurate results. Keeping the same values of TI, TE and thus of FR, $P E E P_{E X T}$ and $V_{T I D}$ and thus PAP with regard to AD_SQUARE, PIP as well as both MAP and MEP are quite the same.

In both AD TRIANG and AD TRAPEZ, PIP resulting from dual-control mode with pre-set $V_{\text {TID }}$ or $V_{\text {MIN }}$, are independent on respiratory resistance and lung compliance, respectively. That is extremely relevant from both physiopathological and clinical point of view since an increase of respiratory resistance (obstructive process) or a reduction of lung compliance (restrictive process) does not affect the maximum value of airways pressure reached for dual-control mode with pre-set $V_{T I D}$ or $V_{\text {MIN }}$, respectively.

So, in conclusion, AD TRAPEZ fits well the requirements for a physiological respiratory pattern concerning endoalveolar pressure and airflow waveforms, while AD_TRIANG exhibits a lower physiological behaviour but is anyhow periodically recommended for performing adequately the powerful diagnostic procedure.

The promising results of the present work establish the rationale for laboratory and clinical test in the field of dual-controlled ventilation with AD_TRAPEZ along with
AD TRIANG

\section{REFERENCES}

[1] Mushin, W.W., Rendell-Backer, L., Thompson, P.W. and Mapleson, W.W. (1980) Automatic ventilation of the lungs. Backwell, Oxford.

[2] Tobin, M.J. (2006) Principles and practice of mechanical ventilation, 2nd Edition, McGraw Hill, New York.

[3] MacIntyre, N.R. and Branson, R.D. (2001) Mechanical ventilation. WB Saunders, Philadelphia.

[4] Chatburn, R.L. (2003) Fundamentals of mechanical ventilation. Mandu Press Ltd, Cleveland Heights.

[5] Branson, R.D. and Chatburn, R.L. (1992) Technical description and classification of modes of ventilator operation. Respiratory Care, 37, 1026-1044.

[6] Chatburn, R.L. and Primiano Jr, F.P. (2001) A new system for understanding modes of mechanical ventilation. Respiratory Care, 46, 604-621.

[7] Chatburn, R.L. (2007) Classification of ventilator modes: Update and proposal for implementation. Respiratory Care, 52, 301-323.

[8] Grianti, F., Montecchia, F., Di Bari, L. and Baldassarri, M. (1996) A versatile mechanical ventilator (DIGIT) with high flow stability and a programmable inspiratory phase flow pattern. IEEE Transactions on Biomedical Engineering, 43, 1062-1072. doi:10.1109/10.541248

[9] Montecchia, F., Guerrisi, M. and Canichella, A. (2007) Advanced lung ventilation system (ALVS) with linear respiratory mechanics assumption for waveform optimization of dual-controlled ventilation. Medical Engineering \& Physics, 29, 259-276. doi:10.1016/j.medengphy.2006.03.006

[10] Campbell, R.S. and Davis, B.R. (2002) Pressure-controlled versus volume-controlled ventilation: Does it matter. Respiratory Care, 47, 416-424.

[11] Taylor, A.E., Render, K.R., Hyatt, R.E. and Parker, J.C. (1989) Clinical respiratory physiology. W. B. Saunders Company, USA.

[12] Hess, D.R. (2005) Ventilator waveform and the physiology of pressure support ventilation. Respiratory Care, 50, 166-186.

[13] MacIntyre, N.R., Gropper, C. and Westfall, T. (1994) Combining pressure-limiting and volume-cycling features in a patient-interactive mechanical breath. Critical Care Medicine, 22, 353-357.

[14] Branson, R.D. and MacIntyre, N.R. (1996) Dual-control modes of mechanical ventilation. Respiratory Care, $\mathbf{4 1}$, 294-305.

[15] Branson, R.D. and Davis Jr, K. (2001) Dual control modes: Combining volume and pressure breaths. Respiratory Care Clinics of North America, 7, 397-408. doi:10.1016/S1078-5337(05)70041-1

[16] Branson, R.D. and Johannigman, J.A. (2005) The role of ventilator graphics when setting dual-controlled modes. Respiratory Care, 50, 187-201.

[17] Laubscher, T.P., Heinrichs, W., Weiler, N., Hartmann, G. and Brunner, J.X. (1994) An adaptive lung ventilation controller. IEEE Transactions on Biomedical Engineering, 41, 51-59. doi:10.1109/10.277271 
[18] Sanborn, W.G. (1993) Microprocessor-based mechanical ventilation. Respiratory Care, 38, 72-109.

[19] Simon, F., Jenayeh, I. and Rake, H.H. (2000) Mechatronics in medical engineering: Advanced control of a ventilation device. Microprocessors and Microsystems, 24, 63-69. doi:10.1016/S0141-9331(99)00068-X

[20] Brunner, J.X. (2001) Principles and history of closedloop controlled ventilation. Respiratory Care Clinics of North America, 7, 341-362.

[21] Branson, R.D., Johannigman, J.A., Campbell, R.S. and Davis Jr., K. (2002) Closed-loop mechanical ventilation. Respiratory Care, 47, 427-451.

[22] Anderson, J.R. and East, T.D. (2002) A closed-loop controller for mechanical ventilation of patients with ARDS. Biomedical Sciences Instrumentation, 38, 289-294.

[23] Tehrani, F.T., Rogers, M., Lo, T., Malinowski, T., Afuwape, S., Lum, M., Grundl, B. and Terry, M. (2004) A dual closed-loop control system for mechanical ventilation. Journal of Clinical Monitoring and Computing, 18, 111-129. doi:10.1023/B:JOCM.0000032744.99885.38

[24] Takeuchi, A., Abe, T., Hirose, M., Kamioka, K., Hamada, A. and Ikeda, N. (2004) Interactive simulation system for artificial ventilation on the internet: Virtual ventilator. Journal of Clinical Monitoring and Computing, 18, 353363. doi:10.1007/s10877-005-6268-0

[25] Chatburn, R.L. (2004) Computer control of mechanical ventilation. Respiratory Care, 49, 507-515.

[26] Tehrani, F.T. and Roum, J.H. (2008) Flex: A new computerized system for mechanical ventilation. Journal of Clinical Monitoring and Computing, 22, 121-130.

[27] Tehrani, F.T. (2008) Automatic control of mechanical ventilation. Part 1: Theory and history of the technology. Journal of Clinical Monitoring and Computing, 22, 409415. doi:10.1007/s10877-008-9150-z

[28] Tehrani, F.T. and Abbasi, S. (2009) Evaluation of a computerized system for mechanical ventilation of infants. Journal of Clinical Monitoring and Computing, 23, 93104. doi:10.1007/s10877-009-9170-3

[29] Barrera, R., Gabovich, N., Sogoloff, H., Groeger, J. and Miodownik, S. (2005) The effect of varying inspiratory flow waveforms on alveolar peak airway pressure and intrinsic positive end expiratory pressure in mechanically ventilated patients. The Internet Journal of Pulmonary Medicine, 5.

[30] Yang, S.C. and Yang, S.P. (2002) Effects of inspiratory flow waveforms on lung mechanics, gas exchange, and respiratory metabolism in COPD patients during mechanical ventilation. Chest, 122, 2096-2104.

[31] Niranjan, S.C., Bidani, A., Ghorbel, F., Zwischenberger, J. B. and Clark Jr, J.W. (1999) Theoretical study of inspiratory flow waveforms during mechanical ventilation on pulmonary blood flow and gas exchange. Computers and Biomedical Research, 32, 355-390.

[32] Lutchen, K.R., Hantos, Z. and Jackson, A.C. (1988) Importance of low-frequency impedance data for reliably quantifying parallel inhomogeneities of respiratory mechanics. IEEE Transactions on Biomedical Engineering, 35, 472-481. doi:10.1109/10.2118

[33] Burke, W.C., Crooke, P.S., Marcy, T.W., Adams, A.B. and Marini, J.J. (1993) Comparison of mathematical and Journal of Applied Physiology, 74, 922-933.

[34] Jonson, B., Beydon, L., Brauer, K., Mansson, C., Valind, S. and Grytzell, H. (1993) Mechanics of respiratory system in healthy anesthetized humans with emphasis on viscoelastic properties. Journal of Applied Physiology, 75, 132-140.

[35] Conti, G., De Blasi, R.A., Lappa, A., Ferretti, A., Antonelli, M., Bufi, M. and Gasparetto, A. (1994) Evaluation of respiratory system resistance in mechanically ventilated patients: The role of the endotracheal tube. Intensive Care Medicine, 20, 421-424.

[36] Crooke, P.S., Hota, S., Marini, J.J. and Hotchkiss, J.R. (2003) Mathematical models of passive, pressure-controlled ventilation with different resistance assumptions. Mathematical and Computer Modelling, 38, 495-502. doi:10.1016/S0895-7177(03)90021-5

[37] Milic-Emili, J., Gottfried, S.B. and Rossi, A. (1987) Noninvasive measurement of respiratory mechanics in ICU patients. International Journal of Clinical Monitoring and Computing, 4, 11-30.

[38] Baconnier, P.F., Carry, P.Y., Eberhard, A., Perdrix, J.P. and Fargnoli, J.M. (1995) A computer program for automatic measurement of respiratory mechanics in artificially ventilated patients. Computer Methods and Program in Biomedicine, 47, 205-220. doi:10.1016/0169-2607(95)01651-9

[39] Lucangelo, U., Bernabè, F. and Blanch, L. (2005) Respiratory mechanics derived from signals in the ventilator circuit. Respiratory Care, 50, 55-65.

[40] Bates, J.H., Hunter, I.W., Sly, P.D., Okubo, S., Filiatrault, S. and Milic-Emili, J. (1987) Effect of valve closure time on the determination of respiratory resistance by flow interruption. Medical and Biological Engineering Computing, 25, 136-140. doi:10.1007/BF02442841

[41] Bates, J.H., Baconnier, P. and Milic-Emili, J. (1988) A theoretical analysis of interrupter technique for measuring respiratory mechanics. Journal of Applied Physiology, 64, c f2204-2214.

[42] Lutchen, K.R., Yang, K., Kaczka, D.W. and Suki, B. (1993) Optimal ventilation waveforms for estimating low -frequency respiratory impedance. Journal of Applied Physiology, 75, 478-488.

[43] Barnas, G.M., Harinath, P., Green, M.D., Suki, B., Kaczka, D.W. and Lutchen, K.R. (1994) Influence of waveform and analysis technique on lung and chest wall properties. Respiratory Physiology, 96, 331-344.

[44] Kaczka, D.W., Ingenito, E.P. and Lutchen, K.R. (1999) Technique to determine inspiratory impedance during mechanical ventilation: Implication for flow limited patients. Annals of Biomedical Engineering, 27, 340-355.

[45] Marini, J.J. and Crooke, P.S. (1993) A general mathematical model for respiratory dynamics relevant to the clinical setting. American Review of Respiratory Disease, 147, 14-24.

[46] Bates, J.H. (2007) A recruitment model of quasi-linear power-law stress adaptation in lung tissue. Annals of Biomedical Engineering, 35, 1165-1174. doi:10.1007/s10439-007-9291-0 